\title{
Chiral violations in domain-wall QCD from one-loop perturbation theory at finite $N_{s}$
}

\author{
Stefano Capitan* \\ Institut für Physik, FB Theoretische Physik \\ Universität Graz, A-8010 Graz, Austria
}

\begin{abstract}
We present perturbative calculations made with domain-wall fermions which possess a finite number of points $N_{s}$ in the extra fifth dimension. We have derived the required propagator functions, investigated the one-loop properties of quark amplitudes at finite $N_{s}$ and evaluated three quantities that can provide insights on chirality-breaking effects from the perturbative side.

First we have computed the residual mass for various choices of $N_{s}$ and of the domain-wall height $M$. We have found that this radiatively induced mass approaches zero reasonably fast with the extent of the fifth dimension, depending on $M$ and on a lesser extent on the coupling $g_{0}$. We have also computed the differences of the renormalization constants of the vector and axial-vector currents and of the scalar and pseudoscalar densities. Finally we have calculated the chirally-forbidden mixing (which at finite $N_{s}$ is suppressed only partially) of an operator which describes the lowest moment of the $g_{2}$ structure function. In general we see that at $M=1.8$, where simulations are usually performed, values of $N_{s}=20$ or larger would be desirable in order for chiral violations to be negligible.

The quantities that we have studied turn out to lose gauge invariance when $N_{s}$ is not infinite. We have also found that anomalous dimensions of operators at finite $N_{s}$ generally depend on $N_{s}$ and $M$. In particular, the vector and axial-vector currents have in general a nonzero anomalous dimension at finite $N_{s}$

PACS numbers: 12.38.Gc,11.30.Rd,11.30.Qc,11.10.Gh
\end{abstract}

\section{INTRODUCTION}

Domain-wall fermions [1, 2, 3] constitute one of the known solutions of the Ginsparg-Wilson relation [4] and are hence invariant with respect to chiral symmetry transformations even away from the continuum limit, for nonvanishing values of the lattice spacing $a$ [5], while avoiding at the same time unpleasant effects like doublers and nonanaliticities. The massless chiral mode generated by the domain wall survives 1-loop renormalization and the theory has been proven to be renormalizable at this order 6 , 7]. The possibility arises of the construction of chiral gauge theories at finite $a$ [8]. Moreover, with this kind of fermions the leading discretization errors are reduced to $O\left(a^{2}\right)$ when chiral symmetry is exact.

A certain amount of chiral symmetry breaking arises however in Monte Carlo simulations of domain-wall fermions, because they must be performed using lattices which have a finite number of points, $N_{s}$, in the fifth dimension. It is only in the theoretical limit in which the extension of the fifth dimension becomes infinite that the chiral modes (which are exponentially confined on the two opposite walls) can fully decouple from each other, yielding an exact chiral symmetry. The chiral modes acquire some mass if the distance between the two walls is not infinite, and to study the magnitude of these chirality-violating effects is one of the main objectives of the present work. With the com-

*stefano.capitani@uni-graz.at puter speeds presently available it is unfortunately not yet possible to obtain significant physics from simulations performed at a large $N_{s}$, where these chiral violations would be numerically negligible. After the first pioneering Monte Carlo implementations of domain-wall fermions 9, 10] and subsequent advances reported in [1, 12, 13, 14, 15] and 16, 17], the most recent simulations, which have considered phenomenological quantities as diverse as weak matrix elements, structure functions and heavy-light meson spectroscopy, have been mostly performed using lattices with only $N_{s}=12$ or 16 (for a selection of the latest results see for example 18, 19, 20, 21, 22, 23, 24, 25, 26, 27, 28, 29, 30, 31]).

The experiences gained in these recent investigations seem to indicate that for extents of the extra fifth dimension as small as $N_{s}=16$ the chirality-breaking effects [14, 32, 33, 34, 35, 36] are still under control. The residual mass $m_{\text {res }}$ for typical lattice spacings of about $a^{-1}=1.5-2 \mathrm{GeV}$ generally turns out to be of $O\left(10^{-3}\right)$ or $O\left(10^{-2}\right)$, depending on whether simulations are carried out in quenched or full QCD, and on which type of gauge action is used 16, 17, 22]. In particular, when using quenched QCD instead of the full theory, or renormalization group improved gauge actions (like Iwasaki and DBW2) instead of the simple plaquette action, the residual mass becomes substantially reduced. This is related to the fact that, for a fixed choice of $a$, the values of $\beta=6 / g_{0}^{2}$ are larger in those cases, and the gauge fields smoother. The residual mass is then in general not very small (expecially in the full QCD case), and it can at times become comparable to the input light sea quark masses. However, if the exponential suppression of 
chirality-breaking effects takes place rather quickly with the length of the fifth dimension, then increasing $N_{s}$ a little further could already be sufficient to obtain at last almost negligible chirality-breaking effects.

The extent to which chiral symmetry is broken in lattices with a small $N_{s}$ is thus one of the most important issues which need to be understood in present domainwall simulations. To the extent that one-loop calculations can provide clues to the true behavior of the truncated domain-wall theory at finite $N_{s}$, we believe that it is useful and interesting to investigate chirality-breaking effects also from the point of view of perturbation theory, complementing nonperturbative investigations of such effects. Towards this end we present here the results of some selected one-loop calculations which we have carried out using the Feynman rules which exactly correspond to the theory at finite $N_{s}$. This is at variance with past domainwall perturbative calculations where, in place of the exact quark propagators, their asymptotic expressions for large $N_{s}$ were instead used [37, 38, 39, 40]. The purpose of this work is to calculate with the exact Feynman rules the deviations from the $N_{s}=\infty$ results in the case where $N_{s}$ is limited to small values of $O(10)$, that is for situations which roughly correspond to present simulations. We use the plaquette gauge action, and we can obtain some estimates of the amount of perturbative chiral violations by focusing on quantities like the additive renormalization to the quark mass (i.e., the residual mass) and the deviations of some amplitudes from their values at $N_{s}=\infty$, including a chirally-forbidden mixing. Since the cost of domain-wall simulations grows approximately linearly with $N_{s}$, it is of some importance to understand how small $N_{s}$ can be kept without occurring in large values of the residual mass. A thorough exploration of large regions in the two-dimensional space spanned by $N_{s}$ and the domain-wall height $M$ would be quite expensive when using Monte Carlo simulations, and perturbation theory remains then often the more practical way for gathering hints of what is happening in this space. The study of the dependence on $M$ and $N_{s}$ of various indicators of chiral violations is thus one of the main aims of the work.

Significant perturbative insights at finite $N_{s}$ beyond tree level have been provided some years ago by Kikukawa, Neuberger and Yamada [41], who diagonalized the mass matrix in the truncated overlap and derived 1-loop equations under certain assumptions on the gauge fields. We improve here on this by providing numerical results for the residual mass. What we present is a complete 1-loop calculation of such radiative effects, with the aim of seeing how $m_{\text {res }}$, and other quantities which can act as indicators of chiral symmetry breaking, behave when $N_{s}$ and $M$ change.

Other methods have also been used to gain insights on these effects. Recently Christ [2] has investigated the residual mass analytically by looking at the eigenfunctions of the five-dimensional transfer matrix [3]. Building on the understanding of the localization properties of the domain-wall modes, characterized by their mobility edge $\lambda_{c}$ [43, 44, 45] (for recent investigations see [46, 47, 48]), the leading effects were estimated as

$$
m_{r e s} \sim R_{e}^{4} \rho_{e}\left(\lambda_{c}\right) \frac{\exp \left(-\lambda_{c} N_{s}\right)}{N_{s}}+R_{l}^{4} \rho_{l}(0) \frac{1}{N_{s}},
$$

where $\rho$ is the density per unit spacetime volume of the eigenvalues of the logarithm of the transfer matrix, and $l$ and $e$ stand for localized and extended modes with average size $R$ respectively. The energy threshold from localized to extended modes is given by the mobility edge $\lambda_{c}$, which is then also responsible for the speed with which the chiral violations decay with $N_{s}$.

This article is organized as follows. In Sect. [1] we introduce all propagator functions which are necessary for the perturbative calculations at finite $N_{s}$, and in Sect. III) we analyze the one-loop renormalization of quark amplitudes in this theory. Since in this article we restrict ourselves to the calculation of finite diagrams, the treatment of divergences in the theory at finite $N_{s}$ is left for a future work. We have however computed the coefficients of the divergent terms in a few cases, and we have seen that they are in general not equal to their continuum values. In fact, they depend on $N_{s}$ and on the height of the domain wall, $M$, as we show in Sect. [V] where we also briefly discuss the implications of this finding. In Sect. $\nabla$ we then present the computation of the residual mass and encounter another feature of calculations at finite $N_{s}$, namely that gauge invariance is lost. In Sect. VI we show the results at finite $N_{s}$ for the difference between the vector and axial-vector renormalization constants, which should be zero at infinite $N_{s}$. In Sect. VIII we finally present a power-divergent mixing due to the breaking of chiral symmetry, for an operator which describes polarized parton distributions, and in Sect. [VIII we discuss what happens near the borders of allowed values of $M$, $M \rightarrow 0$ and $M \rightarrow 2$, before making in Sect. [X] some concluding remarks.

\section{PERTURBATION THEORY}

We work with the standard formulation of domain-wall fermions of Shamir [2],

$$
\begin{aligned}
S_{q}^{D W}= & \sum_{x} \sum_{s=1}^{N_{s}}\left[\frac { 1 } { 2 } \sum _ { \mu } \left(\bar{\psi}_{s}(x)\left(\gamma_{\mu}-r\right) U_{\mu}(x) \psi_{s}(x+\hat{\mu})\right.\right. \\
& \left.-\bar{\psi}_{s}(x)\left(\gamma_{\mu}+r\right) U_{\mu}^{\dagger}(x-\hat{\mu}) \psi_{s}(x-\hat{\mu})\right) \\
& +\left(\bar{\psi}_{s}(x) P_{+} \psi_{s+1}(x)+\bar{\psi}_{s}(x) P_{-} \psi_{s-1}(x)\right) \\
& \left.+(M-1+4 r) \bar{\psi}_{s}(x) \psi_{s}(x)\right] \\
& +m \sum_{x}\left(\bar{\psi}_{N_{s}}(x) P_{+} \psi_{1}(x)+\bar{\psi}_{1}(x) P_{-} \psi_{N_{s}}(x)\right),
\end{aligned}
$$

where we put $r=-1$, that is the Wilson term is added to the action with minus the conventional sign. The height 
of the domain wall, or Dirac mass, $M$, at tree level satisfies $0<M<2$, so that the correct pattern of chiral modes (with no doublers) is attained when $N_{s} \rightarrow \infty$ : in fact for $M<0$ there is no chiral mode, while for $M>2$ there are four of them (and even more when $M$ is further increased). The chiral projectors are $P_{ \pm}=\left(1 \pm \gamma_{5}\right) / 2$. Here and in most of the paper we put $a=1$, but in some contexts, like when discussing the residual mass, the lattice spacing will be explicitly shown.

We refer to [38, 39, 40] for the Feynman rules which derive from this domain-wall action in the limit $N_{s} \rightarrow \infty$. In this article we always work at finite $N_{s}$, and the expressions that we have to use for the quark propagators are then different. They were partially derived in [37], and here we compute the remaining functions and provide the complete set of required propagators.

In (four-dimensional) momentum space the domainwall Dirac operator has the form

$$
\begin{aligned}
D_{s t}(p) & =\delta_{s, t} \sum_{\mu} i \gamma_{\mu} \sin p_{\mu} \\
& +\left(W_{s t}^{+}(p)+m M_{s t}^{+}\right) P_{+}+\left(W_{s t}^{-}(p)+m M_{s t}^{-}\right) P_{-},
\end{aligned}
$$

where the mass matrix is given by

$$
\begin{aligned}
W_{s t}^{ \pm}(p) & =-W(p) \delta_{s, t}+\delta_{s \pm 1, t}, \\
M_{s t}^{+} & =\delta_{s, N_{s}} \delta_{t, 1}, \\
M_{s t}^{-} & =\delta_{s, 1} \delta_{t, N_{s}},
\end{aligned}
$$

and

$$
W(p)=1-M-2 r \sum_{\lambda} \sin ^{2} \frac{p_{\lambda}}{2}
$$

In more explicit form, we have

$$
\begin{aligned}
W^{+}(p) & =\left(\begin{array}{cccc}
-W(p) & 1 & & \\
& -W(p) & \ddots & \\
& & \ddots & 1 \\
& & & -W(p)
\end{array}\right), \\
W^{-}(p) & =\left(\begin{array}{cccc}
-W(p) & & & \\
1 & -W(p) & & \\
& \ddots & \ddots & \\
& & 1 & -W(p)
\end{array}\right), \\
M^{+} & =\left(\begin{array}{c} 
\\
1
\end{array}\right), \\
M^{-} & =\left(\begin{array}{c}
1 \\
1
\end{array}\right) .
\end{aligned}
$$

In practical terms this theory looks like having several flavors of lattice Dirac fermions, which are mixed in a very special way so that a large mass hierarchy is generated. At the end this theory indeed contains one chiral mode which is nearly massless together with $N_{s}-1$ heavy fermions.

In this work we only consider the case in which no explicit mass term appears in the Lagrangian $(m=0)$. The tree-level 5-dimensional quark propagator is then given by

$$
\left\langle\psi_{s}(-p) \bar{\psi}_{t}(p)\right\rangle=\sum_{u}\left[\left(-i \gamma_{\mu} \sin p_{\mu} \delta_{s, u}+W_{s u}^{-}(p)\right) G_{u t}^{R}(p) P_{+}+\left(-i \gamma_{\mu} \sin p_{\mu} \delta_{s, u}+W_{s u}^{+}(p)\right) G_{u t}^{L}(p) P_{-}\right]
$$

where the expressions of the functions $G^{R}(p)$ and $G^{L}(p)$ are [37]

$$
\begin{aligned}
G_{s t}^{R}(p)= & \frac{A(p)}{F(p)}\left[\left(1-W(p) e^{-\alpha(p)}\right)\left(e^{-2 N_{s} \alpha(p)}-1\right) e^{(s+t) \alpha(p)}+2 W(p) \sinh (\alpha(p))\left(e^{(s-t) \alpha(p)}+e^{-(s-t) \alpha(p)}\right)\right. \\
& \left.+\left(1-W(p) e^{\alpha(p)}\right)\left(1-e^{2 N_{s} \alpha(p)}\right) e^{-(s+t) \alpha(p)}\right]+A(p)\left(e^{\left(N_{s}-|s-t|\right) \alpha(p)}+e^{-\left(N_{s}-|s-t|\right) \alpha(p)}\right) \\
G_{s t}^{L}(p)= & \frac{A(p)}{F(p)}\left[\left(e^{-2 \alpha(p)}-W(p) e^{-\alpha(p)}\right)\left(e^{-2 N_{s} \alpha(p)}-1\right) e^{(s+t) \alpha(p)}+2 W(p) \sinh (\alpha(p))\left(e^{(s-t) \alpha(p)}+e^{-(s-t) \alpha(p)}\right)\right. \\
& \left.+\left(e^{2 \alpha(p)}-W(p) e^{\alpha(p)}\right)\left(1-e^{2 N_{s} \alpha(p)}\right) e^{-(s+t) \alpha(p)}\right]+A(p)\left(e^{\left(N_{s}-|s-t|\right) \alpha(p)}+e^{-\left(N_{s}-|s-t|\right) \alpha(p)}\right)
\end{aligned}
$$

In these expressions the quantity $\alpha(p)$ appears, which is

defined by the positive solution of the equation [2, 49]

$$
\cosh (\alpha(p))=\frac{1+W^{2}(p)+\sum_{\lambda} \sin ^{2} p_{\lambda}}{2|W(p)|},
$$


and one uses the abbreviations

$$
\begin{aligned}
A(p)= & \frac{1}{2 W(p) \sinh (\alpha(p))} \frac{1}{2 \sinh \left(N_{s} \alpha(p)\right)}, \\
F(p)= & e^{N_{s} \alpha(p)}\left(1-W(p) e^{\alpha(p)}\right) \\
& -e^{-N_{s} \alpha(p)}\left(1-W(p) e^{-\alpha(p)}\right) .
\end{aligned}
$$

When $W$ is negative, a situation which arises only when $1<M<2$ if the momentum is small enough, the propagator is given by the above equations with the replacements

$$
\begin{gathered}
W \rightarrow-|W|, \\
e^{ \pm \alpha} \rightarrow-e^{ \pm \alpha},
\end{gathered}
$$

which imply that also $\sinh \alpha$ changes sign.

The standard "physical" quark fields used both in Monte Carlo simulations and in perturbative calculations are given by

$$
\begin{aligned}
& q(x)=P_{+} \psi_{1}(x)+P_{-} \psi_{N_{s}}(x) \\
& \bar{q}(x)=\bar{\psi}_{N_{s}}(x) P_{+}+\bar{\psi}_{1}(x) P_{-} .
\end{aligned}
$$

Strictly speaking these fields do not correspond exactly to the chiral modes, which should be instead eigenvectors of the mass matrix, like

$$
\chi_{0}(x)=\sqrt{1-w_{0}^{2}} \sum_{s}\left(P_{+} w_{0}^{s-1} \psi_{s}(x)+P_{-} w_{0}^{N_{s}-s} \psi_{s}(x)\right),
$$

where one calls

$$
w_{0}=W(0)=1-M \text {. }
$$

The physical quark field $q(x)$ is however more convenient to use than $\chi_{0}(x)$, given that $w_{0}$ undergoes already at one loop a renormalization deriving from the additive correction to the domain-wall height $M$ (see Eq. (52) below). Thus one instead takes $q(x)$ to represent the physical zero modes of the theory. Moreover, at finite $N_{s}$ an additional issue about $\chi_{0}(x)$ would arise, because this field is an eigenvector of the mass matrix only up to terms of order $N_{s} e^{-N_{s} \alpha(0)}$ 37, 39, 41].

The computation of matrix elements involving physical states and operators requires the introduction of additional propagators which connect the 4-dimensional physical quark fields with the 5-dimensional quark fields which appear in the Lagrangian. We have here derived the expressions of these propagators for the case of finite $N_{s}$, and they are given by

$$
\begin{aligned}
\left\langle q(-p) \bar{\psi}_{s}(p)\right\rangle= & P_{+}\left\langle\psi_{1}(-p) \bar{\psi}_{s}(p)\right\rangle+P_{-}\left\langle\psi_{N_{s}}(-p) \bar{\psi}_{s}(p)\right\rangle \\
= & \left(\frac{i \gamma_{\mu} \sin p_{\mu}}{E(p)}+e^{-N_{s} \alpha(p)} \frac{2 W(p) \sinh (\alpha(p))}{E(p)\left(1-e^{\left.-2 N_{s} \alpha(p)\right)}\right)}\right. \\
& \quad-\frac{1}{1-e^{-2 N_{s} \alpha(p)}} e^{-\alpha(p)}\left(\left(e^{-(s-1) \alpha(p)}-e^{-2\left(N_{s}-1\right) \alpha(p)} e^{(s-1) \alpha(p)}\right) P_{+}\right. \\
\left\langle\psi_{s}(-p) \bar{q}(p)\right\rangle= & \left\langle\left(e^{-\left(N_{s}-s\right) \alpha(p)}-e^{-2\left(N_{s}-1\right) \alpha(p)} e^{\left(N_{s}-s\right) \alpha(p)}\right) P_{-}\right) \\
= & \left(\left(e^{-\left(N_{s}-s\right) \alpha(p)}-e^{-2 N_{s} \alpha(p)} e^{\left(N_{s}-s\right) \alpha(p)}\right) P_{-}+\left(e^{-(s-1) \alpha(p)}-e^{-2 N_{s} \alpha(p)} e^{(s-1) \alpha(p)}\right) P_{+}\right) \\
& \quad \times\left(\frac{i \gamma_{\mu} \sin p_{\mu}}{E(p)}+e^{-N_{s} \alpha(p)} \frac{2 W(p) \sinh (\alpha(p))}{E(p)\left(1-e^{\left.-2 N_{s} \alpha(p)\right)}\right)}\right) \\
& \quad \frac{1}{1-e^{-2 N_{s} \alpha(p)}} e^{-\alpha(p)}\left(\left(e^{-(s-1) \alpha(p)}-e^{-2\left(N_{s}-1\right) \alpha(p)} e^{(s-1) \alpha(p)}\right) P_{-}\right. \\
& \left.\quad+\left(e^{-\left(N_{s}-s\right) \alpha(p)}-e^{-2\left(N_{s}-1\right) \alpha(p)} e^{\left(N_{s}-s\right) \alpha(p)}\right) P_{+}\right)
\end{aligned}
$$

where we have defined

$$
E(p)=1-W(p) e^{\alpha(p)}-e^{-2 N_{s} \alpha(p)}\left(1-W(p) e^{-\alpha(p)}\right)
$$

We have also numerically checked the above expressions and the correctness of their implementation in our computer codes by verifying the validity for each $s$ (for various choices of $N_{s}$ ) of the identities

$$
G_{1 s}^{L}(p)=G_{s 1}^{L}(p)=-\frac{1}{E(p)}\left(e^{-(s-1) \alpha(p)}-e^{-2 N_{s} \alpha(p)} e^{(s-1) \alpha(p)}\right),
$$




$$
\begin{aligned}
G_{N_{s} s}^{R}(p)=G_{s N_{s}}^{R}(p)= & -\frac{1}{E(p)}\left(e^{-\left(N_{s}-s\right) \alpha(p)}-e^{-2 N_{s} \alpha(p)} e^{\left(N_{s}-s\right) \alpha(p)}\right) \\
\sum_{t} W_{N_{s} t}^{+}(p) G_{t s}^{L}(p)=\sum_{t} W_{s t}^{-}(p) G_{t N_{s}}^{R}(p)= & -\frac{1}{1-e^{-2 N_{s} \alpha(p)}} e^{-\alpha(p)}\left(e^{-\left(N_{s}-s\right) \alpha(p)}-e^{-2\left(N_{s}-1\right) \alpha(p)} e^{\left(N_{s}-s\right) \alpha(p)}\right) \\
& +e^{-N_{s} \alpha(p)} \frac{2 W(p) \sinh (\alpha(p))}{E(p)\left(1-e^{-2 N_{s} \alpha(p)}\right)}\left(e^{-(s-1) \alpha(p)}-e^{-2 N_{s} \alpha(p)} e^{(s-1) \alpha(p)}\right) \\
\sum_{t} W_{1 t}^{-}(p) G_{t s}^{R}(p)=\sum_{t} W_{s t}^{+}(p) G_{t 1}^{L}(p)= & -\frac{1}{1-e^{-2 N_{s} \alpha(p)}} e^{-\alpha(p)}\left(e^{-(s-1) \alpha(p)}-e^{-2\left(N_{s}-1\right) \alpha(p)} e^{(s-1) \alpha(p)}\right) \\
& +e^{-N_{s} \alpha(p)} \frac{2 W(p) \sinh (\alpha(p))}{E(p)\left(1-e^{-2 N_{s} \alpha(p)}\right)}\left(e^{-\left(N_{s}-s\right) \alpha(p)}-e^{-2 N_{s} \alpha(p)} e^{\left(N_{s}-s\right) \alpha(p)}\right),
\end{aligned}
$$

which relate the propagators $\left\langle q(-p) \bar{\psi}_{s}(p)\right\rangle$ and $\left\langle\psi_{s}(-p) \bar{q}(p)\right\rangle$ to $\left\langle\psi_{s}(-p) \bar{\psi}_{t}(p)\right\rangle$.

The calculation of perturbative amplitudes also requires the knowledge of the expressions of these new propagators for small momentum. In this limit we obtain

$$
\begin{aligned}
\left\langle q(-p) \bar{\psi}_{s}(p)\right\rangle_{c}= & -\frac{1-w_{0}^{2}}{1-w_{0}^{2 N_{s}}} \frac{i \not p+w_{0}^{N_{s}}\left(1-w_{0}^{2}\right)}{p^{2}+w_{0}^{2 N_{s}}\left(1-w_{0}^{2}\right)^{2}}\left(\left(w_{0}^{N_{s}-s}-w_{0}^{2 N_{s}} w_{0}^{-\left(N_{s}-s\right)}\right) P_{+}+\left(w_{0}^{s-1}-w_{0}^{2 N_{s}} w_{0}^{-(s-1)}\right) P_{-}\right) \\
& -\frac{1}{1-w_{0}^{2 N_{s}}} w_{0}\left(\left(w_{0}^{s-1}-w_{0}^{2\left(N_{s}-1\right)} w_{0}^{-(s-1)}\right) P_{+}+\left(w_{0}^{N_{s}-s}-w_{0}^{2\left(N_{s}-1\right)} w_{0}^{-\left(N_{s}-s\right)}\right) P_{-}\right) \\
\left\langle\psi_{s}(-p) \bar{q}(p)\right\rangle_{c}= & -\frac{1-w_{0}^{2}}{1-w_{0}^{2 N_{s}}}\left(\left(w_{0}^{N_{s}-s}-w_{0}^{2 N_{s}} w_{0}^{-\left(N_{s}-s\right)}\right) P_{-}+\left(w_{0}^{s-1}-w_{0}^{2 N_{s}} w_{0}^{-(s-1)}\right) P_{+}\right) \frac{i \not p+w_{0}^{N_{s}}\left(1-w_{0}^{2}\right)}{p^{2}+w_{0}^{2 N_{s}}\left(1-w_{0}^{2}\right)^{2}} \\
& -\frac{1}{1-w_{0}^{2 N_{s}}} w_{0}\left(\left(w_{0}^{s-1}-w_{0}^{2\left(N_{s}-1\right)} w_{0}^{-(s-1)}\right) P_{-}+\left(w_{0}^{N_{s}-s}-w_{0}^{2\left(N_{s}-1\right)} w_{0}^{-\left(N_{s}-s\right)}\right) P_{+}\right)
\end{aligned}
$$

where $w_{0}$ is defined in Eq. (23). Since $w_{0}=e^{-\alpha(0)}$, it is easy to see that the terms which are proportional to $w_{0}^{N_{s}}=e^{-N_{s} \alpha(0)}$ rapidly approach zero when $N_{s}$ becomes large. In the derivation of the above formulae we have used the useful small momentum expansions

$$
\begin{aligned}
1-W(p) e^{\alpha(p)} & =-\frac{p^{2}}{1-w_{0}^{2}} \\
1-W(p) e^{-\alpha(p)} & =1-w_{0}^{2}-w_{0} \frac{1-w_{0}-w_{0}^{2}}{1-w_{0}^{2}} p^{2}
\end{aligned}
$$

and

$$
e^{-N_{s} \alpha(p)} \frac{2 W(p) \sinh (\alpha(p))}{E(p)}=-\frac{w_{0}^{N_{s}}\left(1-w_{0}^{2}\right)^{2}}{p^{2}+w_{0}^{2 N_{s}}\left(1-w_{0}^{2}\right)^{2}}
$$

and we have dropped all terms of order $p^{2} w_{0}^{N_{s}}$ and higher, which are much smaller than either of the factors $p^{2}$ or $w_{0}^{N_{s}}$ alone, and are not relevant when $p \rightarrow 0$. It is easy to check that all the propagators that we have derived in this Section reduce to the expressions used in the calculations of Refs. [38, 39, 40] when $N_{s}$ is large. Notice also that the function $E(p)$ introduced here tends in this approximation to the function $F(p)$ as defined in those articles. The above expressions for the small momentum propagators can also be used for $M>1$ without further modifications (as we have also numerically checked).

Finally, we also need the function that describes the propagation of the physical fields alone. This is given by

$$
\begin{array}{r}
\langle q(-p) \bar{q}(p)\rangle=\frac{1}{E(p)}\left(i \gamma_{\mu} \sin p_{\mu}\left(1-e^{-2 N_{s} \alpha(p)}\right)\right. \\
\left.+e^{-N_{s} \alpha(p)} \cdot 2 W(p) \sinh (\alpha(p))\right),
\end{array}
$$

which in the limit of small momentum becomes

$$
\langle q(-p) \bar{q}(p)\rangle_{c}=-\left(1-w_{0}^{2}\right) \frac{i \not p+w_{0}^{N_{s}}\left(1-w_{0}^{2}\right)}{p^{2}+w_{0}^{2 N_{s}}\left(1-w_{0}^{2}\right)^{2}} .
$$

It is interesting to see that it is also possible to calculate $\langle q(-p) \bar{q}(p)\rangle$ in an alternative way directly from the 5dimensional propagator of Eq. (12):

$$
\begin{aligned}
\langle q \bar{q}\rangle= & P_{+}\left\langle\psi_{1} \bar{\psi}_{N_{s}}\right\rangle P_{+}+P_{+}\left\langle\psi_{1} \bar{\psi}_{1}\right\rangle P_{-} \\
& +P_{-}\left\langle\psi_{N_{s}} \bar{\psi}_{N_{s}}\right\rangle P_{+}+P_{-}\left\langle\psi_{N_{s}} \bar{\psi}_{1}\right\rangle P_{-} \\
= & -\frac{1}{2}\left[i \gamma_{\mu} \sin p_{\mu}\left(G_{N_{s} N_{s}}^{R} P_{+}+G_{11}^{L} P_{-}\right)\right. \\
& \left.+W\left(G_{1 N_{s}}^{R} P_{+}+G_{N_{s} 1}^{L} P_{-}\right)\right] .
\end{aligned}
$$

The fact that in this way we obtain again the result of Eq. (37) provides a good check of the above formulae.

Domain-wall fermions present thus at finite $N_{s}$ some new peculiar features. Although the theory which we have started from is described by a Lagrangian of massless quarks, the propagator of the physical quark field, 
$\langle q(-p) \bar{q}(p)\rangle_{c}$, acquires when $N_{s}$ is kept finite a nonvanishing mass term, which at tree level is given by

$$
a m_{\text {res }}^{(0)}=-w_{0}^{N_{s}}\left(1-w_{0}^{2}\right)=-(1-M)^{N_{s}} M(2-M),
$$

as can be seen from the general expression of a fermion propagator of mass $\mu$ for small momentum in Euclidean space:

$$
\frac{-i \not p+\mu}{p^{2}+\mu^{2}}=\frac{1}{i \not p+\mu} .
$$

We readily see that this tree-level residual mass vanishes when $N_{s}=\infty$. We will only consider even values of $N_{s}$, in which case the fermion determinant can be proven to be positive (so that the square root of the two-flavor theory is well defined and an odd number of dynamical flavors can be simulated). Then $m_{\text {res }}^{(0)}$ is always a negative quantity. With our calculation we have thus reproduced, up to a sign, the result for $m_{\text {res }}^{(0)}$ found in [2, 32, 33, 39, 41]. This was derived by considering the quadratic operator $D^{\dagger} D$, which could perhaps explain the sign discrepancy.

We will see that when the one-loop corrections are taken into account, the residual mass changes sign and becomes positive.

If we had used the chiral mode $\chi_{0}$, Eq. (22), the propagator for small momentum would have been given by

$$
\begin{aligned}
\left\langle\left(1-w_{0}^{2}\right) \chi_{0} \bar{\chi}_{0}\right\rangle_{c}= & -\frac{1-w_{0}^{2}}{p^{2}+w_{0}^{2 N_{s}}\left(1-w_{0}^{2}\right)^{2}} \\
& \times\left[i \not p+w_{0}^{N_{s}}\left(1-w_{0}^{2}\right)\left(1-2 w_{0}^{2 N_{s}}\right)\right. \\
& \left.\quad-N_{s} \frac{w_{0}^{2 N_{s}}\left(1-w_{0}^{2}\right)^{2}}{1-w_{0}^{2 N_{s}}}\right],
\end{aligned}
$$

which compared to $\langle q \bar{q}\rangle_{c}$ has correction terms of higher order in $w_{0}^{N_{s}}$ in the numerator. We remind however that $\chi_{0}$ deviates from what would be the exact chiral mode for finite $N_{s}$ by terms of order $N_{s} w_{0}^{N_{s}}$. The real physical propagator would then also present further corrections terms, and it could be that the expression in Eq. (41) would get simpler.

To complete the setup of our calculations, we also recall that we use the plaquette action in a general covariant gauge, where the gluon propagator is given by

$$
G_{\mu \nu}(k)=\frac{1}{4 \sum_{\rho} \sin ^{2} \frac{k_{\rho}}{2}}\left(\delta_{\mu \nu}-(1-\alpha) \frac{4 \sin \frac{k_{\mu}}{2} \sin \frac{k_{\nu}}{2}}{4 \sum_{\lambda} \sin ^{2} \frac{k_{\lambda}}{2}}\right),
$$

so that $\alpha=1$ and $\alpha=0$ correspond to the Feynman and Landau gauges respectively. The QCD vertices that we need are the usual ones, and (apart from color factors) they have the form

$$
\begin{aligned}
V_{\mu}^{(1)}(p) & =-g_{0}\left(i \gamma_{\mu} \cos \frac{p_{\mu}}{2}-\sin \frac{p_{\mu}}{2}\right) \\
V_{\mu \nu}^{(2)}(p) & =\frac{1}{2} g_{0}^{2}\left(i \gamma_{\mu} \sin \frac{p_{\mu}}{2}+\cos \frac{p_{\mu}}{2}\right) \cdot \delta_{\mu \nu}
\end{aligned}
$$

for the interaction of the quark current with one gluon and two gluons respectively, where $p$ in this case stands for the sum of the incoming and outgoing quark momenta.

\section{RENORMALIZATION AT FINITE $N_{s}$}

Let us now investigate the properties of the renormalization of the self-energy of a massless quark at finite $N_{s}$. We first notice that the one-loop propagator of the physical field can be written, according to the general form of the external legs $\left\langle q(-p) \bar{\psi}_{s}(p)\right\rangle_{c}$ and $\left\langle\psi_{s}(-p) \bar{q}(p)\right\rangle_{c}$, as

$$
\begin{aligned}
\langle q(-p) \bar{q}(p)\rangle_{1 \text { loop }} & =\frac{1-w_{0}^{2}}{i \not p-w_{0}^{N_{s}}\left(1-w_{0}^{2}\right)}+\frac{1-w_{0}^{2}}{i \not p-w_{0}^{N_{s}}\left(1-w_{0}^{2}\right)} \Sigma_{q}(p) \frac{1-w_{0}^{2}}{i \not p-w_{0}^{N_{s}}\left(1-w_{0}^{2}\right)} \\
& =\frac{1-w_{0}^{2}}{i \not p-w_{0}^{N_{s}}\left(1-w_{0}^{2}\right)-\left(1-w_{0}^{2}\right) \Sigma_{q}(p)},
\end{aligned}
$$

where

$$
\begin{aligned}
\Sigma_{q}(p)=\sum_{s, t} & \frac{1}{1-w_{0}^{2 N_{s}}}\left[\left(w_{0}^{N_{s}-s}-w_{0}^{2 N_{s}} w_{0}^{-\left(N_{s}-s\right)}\right) P_{+}+\left(w_{0}^{s-1}-w_{0}^{2 N_{s}} w_{0}^{-(s-1)}\right) P_{-}\right. \\
& \left.-w_{0} \frac{i \not p-w_{0}^{N_{s}}\left(1-w_{0}^{2}\right)}{1-w_{0}^{2}}\left(\left(w_{0}^{s-1}-w_{0}^{2\left(N_{s}-1\right)} w_{0}^{-(s-1)}\right) P_{+}+\left(w_{0}^{N_{s}-s}-w_{0}^{2\left(N_{s}-1\right)} w_{0}^{-\left(N_{s}-s\right)}\right) P_{-}\right)\right] \\
& \cdot \Sigma_{s t}(p) \\
& \cdot \frac{1}{1-w_{0}^{2 N_{s}}}\left[\left(w_{0}^{N_{s}-t}-w_{0}^{2 N_{s}} w_{0}^{-\left(N_{s}-t\right)}\right) P_{-}+\left(w_{0}^{t-1}-w_{0}^{2 N_{s}} w_{0}^{-(t-1)}\right) P_{+}\right.
\end{aligned}
$$




$$
\left.-w_{0}\left(\left(w_{0}^{t-1}-w_{0}^{2\left(N_{s}-1\right)} w_{0}^{-(t-1)}\right) P_{-}+\left(w_{0}^{N_{s}-t}-w_{0}^{2\left(N_{s}-1\right)} w_{0}^{-\left(N_{s}-t\right)}\right) P_{+}\right) \frac{i \not p-w_{0}^{N_{s}}\left(1-w_{0}^{2}\right)}{1-w_{0}^{2}}\right]
$$

contributes to the $g_{0}^{2}$ order and the functions $G_{s t}^{R}$ and $G_{s t}^{L}$ give contributions to $\Sigma_{s t}(p)$ only. The general form of $\Sigma_{q}(p)$ is

$$
\Sigma_{q}(p)=\frac{\bar{g}^{2}}{1-w_{0}^{2}}\left[\frac{\Sigma_{0}}{a}+i \not p\left(c_{\Sigma_{1}}^{\left(N_{s}, M\right)} \log a^{2} p^{2}+\Sigma_{1}\right)-\left(i \not p-w_{0}^{N_{s}}\left(1-w_{0}^{2}\right)\right) \frac{2 w_{0}}{1-w_{0}^{2}} \Sigma_{3}\right]
$$

where from now on we call for brevity $\bar{g}^{2}=\left(g_{0}^{2} / 16 \pi^{2}\right) C_{F}$ (with $C_{F}=\left(N_{c}^{2}-1\right) / 2 N_{c}$ for the $S U\left(N_{c}\right)$ gauge group). We can observe that $\Sigma_{q}$ differs in a few aspects from the corresponding expression for infinite $N_{s}$. In fact, apart from the different coefficient of the logarithmic term, which now depends on $N_{s}$ and $M$ (see Section [V]), and the slightly different coefficient of $\Sigma_{3}$, it also contains a totally new contribution proportional to $1 / a$, called $\Sigma_{0}$, which is a mass correction term. The one-loop radiatively induced mass is indeed given by

$$
a m_{r e s}^{(1)}=-w_{0}^{N_{s}}\left(1-w_{0}^{2}\right)-\bar{g}^{2} \Sigma_{0}
$$

as can be easily seen when the one-loop correction to the quark propagator is cast in the same form as its tree-level expression:

$$
\begin{aligned}
\langle q(-p) \bar{q}(p)\rangle_{1 \text { loop }} & =\frac{1-w_{0}^{2}}{i \not p-w_{0}^{N_{s}}\left(1-w_{0}^{2}\right)-\left(1-w_{0}^{2}\right) \Sigma_{q}(p)} \\
& =\frac{1-w_{0}^{2}}{i \not p Z_{2}^{-1}+m_{\text {res }}^{(1)}} Z_{w} .
\end{aligned}
$$

This $O\left(a^{-1}\right)$ critical mass, reminiscent of the analogous quantity for Wilson fermions, vanishes when the theory describes exact chiral fermions, that is at infinite $N_{s}$, but is different from zero when computations are done at any finite $N_{s}$. This means that in the latter case the $\Sigma_{0}$ contribution generates a finite additive renormalization to the quark mass, which can represent a measure of chirality-breaking effects. We associate this perturbative critical mass $m_{r e s}$ with the residual mass which in Monte Carlo simulations is computed by looking at the explicit chiral symmetry breaking term in the axial Ward identities.

The results above should not come as a surprise, since after all we are working here with a theory of $N_{s}$ Wilson fermions, $N_{s}-1$ of which are heavy states (not counting the doublers), and the necessity of a fine tuning to some critical mass comes out naturally. As in the case of Wilson fermions, where the hopping parameter is renormalized away from its tree level value $1 / 8$, in order to obtain a massless pion in domain-wall simulations the quark mass must be tuned to a nonzero number, which at one loop is given by the critical mass given above. This defines the chiral limit when no explicit mass term appears in the Lagrangian. Of course higher loops and nonperturbative effects give further contributions to the shift of the critical mass. From a practical point of view, it is interesting to see how small this critical mass is, together with its dependence on $N_{s}$ (and $M$ ). In the free case the numerical results for $m_{\text {res }}$ according to Eq. (39) are collected in Table $\amalg$ (where they have already been multiplied for $16 \pi^{2}$, so as to ease the comparisons with the one-loop results for the critical mass presented in the next Section). We expect that the critical mass substantially decreases when $N_{s}$ becomes large, as can be verified at one loop from Tables XII to XV] in the next Section.

The other quantities appearing in the last line of Eq. (49) are

$$
Z_{2}=1+\bar{g}^{2}\left(c_{\Sigma_{1}}^{\left(N_{s}, M\right)} \log a^{2} p^{2}+\Sigma_{1}\right)
$$

which is the quark wave function renormalization factor, and

$$
Z_{w}=1-\frac{2 w_{0}}{1-w_{0}^{2}} \bar{g}^{2} \Sigma_{3}=1+\bar{g}^{2} z_{w}
$$

which represents an additive renormalization to $w_{0}$ and hence to the domain-wall height $M$ [38], as can be inferred from

$$
\left(1-w_{0}^{2}\right) Z_{w}=1-\left(w_{0}+\bar{g}^{2} \Sigma_{3}\right)^{2}+O\left(\bar{g}^{4}\right) .
$$

There is indeed no chiral symmetry which can protect this mass, even at $N_{s}=\infty$. The additive renormalization to $M$ can be traced back, in the damping factors $\left\langle q(-p) \bar{\psi}_{s}(p)\right\rangle_{c}$ and $\left\langle\psi_{s}(-p) \bar{q}(p)\right\rangle_{c}$ in Eq. (46), to the terms which are proportional to $i \not p$. Notice how these terms are also proportional to $w_{0}^{N_{s}}\left(1-w_{0}^{2}\right)$, which although being of a different order in $a$ is required for the correct recasting of the one-loop propagators in the form of Eq. (49).

The renormalization of a composite operator $\bar{q}(x) O q(x)$ which is multiplicatively renormalizable can also be expressed in a simple way. Again, by looking at the general form of the propagators the one-loop matrix element of such an operator between "physical" quark states can be written as

$$
\begin{aligned}
\langle(\bar{q} O q) q \bar{q}\rangle_{1 \text { loop }}= & \frac{1-w_{0}^{2}}{i \not p-w_{0}^{N_{s}}\left(1-w_{0}^{2}\right)} \cdot A_{O}(p) \cdot O \\
& \times \frac{1-w_{0}^{2}}{i \not p-w_{0}^{N_{s}}\left(1-w_{0}^{2}\right)},
\end{aligned}
$$

where $A_{O}(p)$ contains the contribution of the damping factors. For a logarithmically divergent operator it takes 
the form

$$
A_{O}(p)=\bar{g}^{2}\left(-\gamma_{O}^{\left(N_{s}, M\right)} \log a^{2} p^{2}+B_{O}\right),
$$

where the anomalous dimension turns out in general to be a function of $N_{s}$ and $M$, even at lowest order.

\section{DIVERGENCES AT FINITE $N_{s}$}

As we have anticipated in the previous Section, in the case of the self-energy, Eq. (47), and of a divergent op- erator, Eq. (54), the coefficients of the logarithmic divergences turn out to depend on $N_{s}$ and $M$. It is only when $N_{s}=\infty$ that they become equal to the ones calculated in the continuum. We give here some examples of this phenomenon by computing a few of these coefficients.

The divergence of the half-circle diagram of the selfenergy comes from the terms which are of first order in $p$ in the integral

$$
\begin{aligned}
2 i \int \frac{d^{4} k}{(2 \pi)^{4}} \frac{1-w_{0}^{2}}{\left(1-w_{0}^{2 N_{s}}\right)^{2}} \sum_{\rho \lambda} \sum_{s t} & \left(\left(w_{0}^{N_{s}-s}-w_{0}^{2 N_{s}} w_{0}^{-\left(N_{s}-s\right)}\right) P_{+}+\left(w_{0}^{s-1}-w_{0}^{2 N_{s}} w_{0}^{-(s-1)}\right) P_{-}\right) \\
& \times \frac{k \cdot p}{(k-p)^{4}}\left(\delta_{\rho \lambda}-(1-\alpha) \frac{k_{\rho} k_{\lambda}}{(k-p)^{2}}\right) \cdot \gamma_{\rho} \cdot \gamma_{\mu} k_{\mu} \cdot \gamma_{\lambda} \cdot\left(\widetilde{G}_{s t}^{R}(k) P_{+}+\widetilde{G}_{s t}^{L}(k) P_{-}\right) \\
& \times\left(\left(w_{0}^{N_{s}-t}-w_{0}^{2 N_{s}} w_{0}^{-\left(N_{s}-t\right)}\right) P_{-}+\left(w_{0}^{t-1}-w_{0}^{2 N_{s}} w_{0}^{-(t-1)}\right) P_{+}\right)
\end{aligned}
$$

where the small $k$ expansions of the functions $G^{R}$ and $G^{L}$ are given by

$$
\begin{aligned}
& \widetilde{G}_{s t}^{R}(k)=\frac{1-w_{0}^{2}}{k^{2}+\left(m_{\text {res }}^{(0)}\right)^{2}}\left(w_{0}^{2 N_{s}-s-t}-\frac{w_{0}^{2 N_{s}}}{1-w_{0}^{2 N_{s}}}\left(w_{0}^{s-t}+w_{0}^{-(s-t)}\right)+O\left(k^{2}\right)\right), \\
& \widetilde{G}_{s t}^{L}(k)=\frac{1-w_{0}^{2}}{k^{2}+\left(m_{\text {res }}^{(0)}\right)^{2}}\left(w_{0}^{s+t-2}-\frac{w_{0}^{2 N_{s}}}{1-w_{0}^{2 N_{s}}}\left(w_{0}^{s-t}+w_{0}^{-(s-t)}\right)+O\left(k^{2}\right)\right),
\end{aligned}
$$

The terms proportional to $w_{0}^{2 N_{s}-s-t}$ and $w_{0}^{s+t-2}$, which are localized near the two walls and provide the leading approximation for large $N_{s}$ to the continuum coefficient, have already been given in [37, 38].

After doing the gamma algebra and carrying out the sums in the indices $s$ and $t$ in the fifth dimension, we get a compact analytic expression for the coefficient of the logarithmic term, as a function of $N_{s}$ and $M$ :

$$
c_{\Sigma_{1}}^{\left(N_{s}, M\right)}=c_{\Sigma_{1}}^{\infty} \cdot\left(1-N_{s} w_{0}^{2 N_{s}} \frac{1-w_{0}^{2}}{1-w_{0}^{2 N_{s}}}\right)\left(1-N_{s} w_{0}^{2 N_{s}} \frac{1-w_{0}^{2}}{1-w_{0}^{2 N_{s}}}+2 \frac{w_{0}^{2\left(N_{s}+1\right)}}{1-w_{0}^{2 N_{s}}}\left(1-N_{s} \frac{1-w_{0}^{2}}{w_{0}^{2}\left(1-w_{0}^{2 N_{s}}\right)}\right)\right)
$$

where $c_{\Sigma_{1}}^{\infty}=\alpha$ is the value of the coefficient in the case of exact chiral symmetry. Numerical values of $c_{\Sigma_{1}}^{\left(N_{s}, M\right)}$ for various choices of $N_{s}$ and $M$ in Feynman gauge are shown in Table II

For a generic bilinear $\bar{q}(x) \Gamma q(x)$, the divergence is obtained by computing the following integral from the vertex diagram:

$$
\begin{aligned}
& -\int \frac{d^{4} k}{(2 \pi)^{4}} \frac{\left(1-w_{0}^{2}\right)^{2}}{\left(1-w_{0}^{2 N_{s}}\right)^{4}} \sum_{\rho \lambda} \sum_{s t}\left(\left(w_{0}^{N_{s}-s}-w_{0}^{2 N_{s}} w_{0}^{-\left(N_{s}-s\right)}\right) P_{+}+\left(w_{0}^{s-1}-w_{0}^{2 N_{s}} w_{0}^{-(s-1)}\right) P_{-}\right. \\
& \left.+w_{0}^{N_{s}+1}\left(\left(w_{0}^{s-1}-w_{0}^{2\left(N_{s}-1\right)} w_{0}^{-(s-1)}\right) P_{+}+\left(w_{0}^{N_{s}-s}-w_{0}^{2\left(N_{s}-1\right)} w_{0}^{-\left(N_{s}-s\right)}\right) P_{-}\right)\right) \\
& \times \gamma_{\rho}\left[\left(\left(w_{0}^{N_{s}-s}-w_{0}^{2 N_{s}} w_{0}^{-\left(N_{s}-s\right)}\right) P_{-}+\left(w_{0}^{s-1}-w_{0}^{2 N_{s}} w_{0}^{-(s-1)}\right) P_{+}\right) \frac{i \not k-m_{\text {res }}^{(0)}}{k^{2}+\left(m_{\text {res }}^{(0)}\right)^{2}}\right. \\
& \left.+\frac{w_{0}}{1-w_{0}^{2}}\left(\left(w_{0}^{s-1}-w_{0}^{2\left(N_{s}-1\right)} w_{0}^{-(s-1)}\right) P_{-}+\left(w_{0}^{N_{s}-s}-w_{0}^{2\left(N_{s}-1\right)} w_{0}^{-\left(N_{s}-s\right)}\right) P_{+}\right)\right] \\
& \times \Gamma \cdot \frac{1}{(k-p)^{2}}\left(\delta_{\rho \lambda}-(1-\alpha) \frac{k_{\rho} k_{\lambda}}{(k-p)^{2}}\right) \\
& \times\left[\frac{i \not k-m_{\text {res }}^{(0)}}{k^{2}+\left(m_{\text {res }}^{(0)}\right)^{2}}\left(\left(w_{0}^{N_{s}-t}-w_{0}^{2 N_{s}} w_{0}^{-\left(N_{s}-t\right)}\right) P_{+}+\left(w_{0}^{t-1}-w_{0}^{2 N_{s}} w_{0}^{-(t-1)}\right) P_{-}\right)\right.
\end{aligned}
$$




$$
\begin{aligned}
& \left.+\frac{w_{0}}{1-w_{0}^{2}}\left(\left(w_{0}^{t-1}-w_{0}^{2\left(N_{s}-1\right)} w_{0}^{-(t-1)}\right) P_{-}+\left(w_{0}^{N_{s}-t}-w_{0}^{2\left(N_{s}-1\right)} w_{0}^{-\left(N_{s}-t\right)}\right) P_{+}\right)\right] \\
\times & \gamma_{\lambda}\left(\left(w_{0}^{N_{s}-t}-w_{0}^{2 N_{s}} w_{0}^{-\left(N_{s}-t\right)}\right) P_{-}+\left(w_{0}^{t-1}-w_{0}^{2 N_{s}} w_{0}^{-(t-1)}\right) P_{+}\right. \\
& \left.+w_{0}^{N_{s}+1}\left(\left(w_{0}^{t-1}-w_{0}^{2\left(N_{s}-1\right)} w_{0}^{-(t-1)}\right) P_{-}+\left(w_{0}^{N_{s}-t}-w_{0}^{2\left(N_{s}-1\right)} w_{0}^{-\left(N_{s}-t\right)}\right) P_{+}\right)\right) .
\end{aligned}
$$

The coefficient of the logarithmic term turns out after many simplifications to be given by

$$
c_{\Gamma}^{\left(N_{s}, M\right)}=c_{\Gamma}^{\infty} \cdot\left(1-N_{s} w_{0}^{2 N_{s}} \frac{1-w_{0}^{2}}{1-w_{0}^{2 N_{s}}}\right)\left(1-N_{s} w_{0}^{2 N_{s}} \frac{1-w_{0}^{2}}{1-w_{0}^{2 N_{s}}}-4 w_{0}^{2 N_{s}}\left(1-N_{s} \frac{1-w_{0}^{2}}{1-w_{0}^{2 N_{s}}}\right)\right),
$$

where $c_{\Gamma}^{\infty}$ is its continuum value, that is $c_{S}^{\infty}=c_{P}^{\infty}=-3+\alpha, c_{V}^{\infty}=c_{A}^{\infty}=-\alpha$ and $c_{T}^{\infty}=1-\alpha$. The dependence on $N_{s}$ and $M$ is different from the one of the self-energy, and thus we find that the anomalous dimensions of all bilinears must also depend on these parameters. Numerical values of $c_{V}^{\left(N_{s}, M\right)}$ are reported in Table III for the Feynman gauge.

A remarkable consequence of the above formulae is that the anomalous dimension of the vector and axial-vector currents does not vanish anymore. The contributions coming from the half-circle diagram of the self-energy and from the vertex diagram of the vector (or axial-vector) current do not compensate each other, because part of the subleading terms are different. In Feynman gauge one gets

$$
\gamma_{V}^{\left(N_{s}, M\right)}=2 w_{0}^{2 N_{s}} \cdot\left(1-N_{s} w_{0}^{2 N_{s}} \frac{1-w_{0}^{2}}{1-w_{0}^{2 N_{s}}}\right)\left(N_{s} \frac{1-w_{0}^{2}}{1-w_{0}^{2 N_{s}}}\left(2+\frac{1}{1-w_{0}^{2 N_{s}}}\right)-2-\frac{w_{0}^{2}}{1-w_{0}^{2 N_{s}}}\right),
$$

and the numerical values of this anomalous dimension are reported in Table IV] These currents have then for any finite $N_{s}$ an anomalous dimension which is different from zero. Only in the Landau gauge it is equal to the case of exact chiral symmetry and thus vanishes, but this happens just because the coefficients of the logarithms, being proportional to their values at $N_{s}=\infty$, in this gauge vanish separately for the self-energy and for these currents.

The deviations of the anomalous dimension of the scalar and pseudoscalar densities from its $N_{s}=\infty$ value are given for the Feynman gauge in Table $\nabla$

Thus, in domain-wall fermions for any finite $N_{s}$ already at the one-loop level the anomalous dimensions of the continuum and lattice versions of an operator are not the same, and this raises some issues about the correct procedure with which one must carry out the matching of lattice operators to a continuum scheme in this case. In fact, the matching formula [50, 51]

$$
\frac{\left\langle q\left|O^{\overline{\mathrm{MS}}}\right| q\right\rangle}{\left\langle q\left|O^{l a t}\right| q\right\rangle}=1-\bar{g}^{2}\left(-\gamma_{O} \log a^{2} \mu^{2}+R^{l a t}-R^{\overline{\mathrm{MS}}}\right)
$$

is only valid provided the coefficients of the logarithmic terms in

$$
\left\langle q\left|O^{l a t}\right| q\right\rangle=\left(1+\bar{g}^{2}\left(-\gamma_{O} \log a^{2} p^{2}+R^{l a t}\right)\right) \cdot\left\langle q\left|O^{\text {tree }}\right| q\right\rangle
$$

and

$$
\left\langle q\left|O^{\overline{\mathrm{MS}}}\right| q\right\rangle=\left(1+\bar{g}_{\overline{\mathrm{MS}}}^{2}\left(-\gamma_{O} \log \frac{p^{2}}{\mu^{2}}+R^{\overline{\mathrm{MS}}}\right)\right) \cdot\left\langle q\left|O^{\text {tree }}\right| q\right\rangle
$$

are the same. But this does not happen for domain-wall fermions at finite $N_{s}$, because the $\gamma_{O}$ in Eq. (63) must be replaced with a $\gamma_{O}^{N_{s}, M}$ which depends on $N_{s}$, and thus this logarithmic term cannot be combined with the one of Eq. (64), whose coefficient is given by the continuum theory. In particular, the $p$ dependence cannot be eliminated from the matching formula.

It could well be that all this is connected to the fact that the domain-wall theory at finite $N_{s}$ is loaded with some pathologies, as it possesses no analytical AtiyahSinger index. It is indeed well known that in all formulations of chiral fermions that recover the correct quantum anomalies one needs (in one way or another) an infinite number of fermion fields. With a finite number of these fields what happens is that either the two chiralities cannot be completely separated or that the right anomalies cannot be reproduced. In domain-wall at finite $N_{s}$, which corresponds to a finite number of fermions fields, we could then anticipate something like this to happen. We will see in the following Section that in the theory truncated at finite $N_{s}$ this is not the only strange feature which arises, but that gauge invariance is lost as well, even for finite quantities.

\section{RESIDUAL MASS}

In this Section we report, for several choices of $N_{s}$ (and $M)$, the results that we have obtained for $\Sigma_{0}$. This quantity enters into the description of the one-loop quark selfenergy, Eq. (47), and determines the critical (or residual) mass at this level. The numbers that we have obtained for $\Sigma_{0}$ are valid both in the quenched and unquenched 
TABLE I: Residual mass at tree level in lattice units (multiplied for $16 \pi^{2}$ ).

\begin{tabular}{|c|c|c|c|c|c|c|c|c|c|}
\hline$M$ & $N_{s}=8$ & $N_{s}=12$ & $N_{s}=16$ & $N_{s}=20$ & $N_{s}=24$ & $N_{s}=28$ & $N_{s}=32$ & $N_{s}=48$ & $N_{s}=\infty$ \\
\hline 0.1 & -12.91556 & -8.47390 & -5.55973 & -3.64774 & -2.39328 & -1.57023 & -1.03023 & -0.19090 & 0 \\
\hline 0.2 & -9.53767 & -3.90663 & -1.60015 & -0.65542 & -0.26846 & -0.10996 & -0.04504 & -0.00127 & 0 \\
\hline 0.3 & -4.64274 & -1.11472 & -0.26764 & -0.06426 & -0.01543 & -0.00370 & -0.00089 & 0.00000 & 0 \\
\hline 0.4 & -1.69750 & -0.22000 & -0.02851 & -0.00370 & -0.00048 & -0.00006 & -0.00001 & 0.00000 & 0 \\
\hline 0.5 & -0.46264 & -0.02891 & -0.00181 & -0.00011 & -0.00001 & 0.00000 & 0.00000 & 0.00000 & 0 \\
\hline 0.6 & -0.08693 & -0.00223 & -0.00006 & 0.00000 & 0.00000 & 0.00000 & 0.00000 & 0.00000 & 0 \\
\hline 0.7 & -0.00943 & -0.00008 & 0.00000 & 0.00000 & 0.00000 & 0.00000 & 0.00000 & 0.00000 & 0 \\
\hline 0.8 & -0.00039 & 0.00000 & 0.00000 & 0.00000 & 0.00000 & 0.00000 & 0.00000 & 0.00000 & 0 \\
\hline 0.9 & 0.00000 & 0.00000 & 0.00000 & 0.00000 & 0.00000 & 0.00000 & 0.00000 & 0.00000 & 0 \\
\hline 1.0 & 0.00000 & 0.00000 & 0.00000 & 0.00000 & 0.00000 & 0.00000 & 0.00000 & 0.00000 & 0 \\
\hline 1.1 & 0.00000 & 0.00000 & 0.00000 & 0.00000 & 0.00000 & 0.00000 & 0.00000 & 0.00000 & 0 \\
\hline 1.2 & -0.00039 & 0.00000 & 0.00000 & 0.00000 & 0.00000 & 0.00000 & 0.00000 & 0.00000 & 0 \\
\hline 1.3 & -0.00943 & -0.00008 & 0.00000 & 0.00000 & 0.00000 & 0.00000 & 0.00000 & 0.00000 & 0 \\
\hline 1.4 & -0.08693 & -0.00223 & -0.00006 & 0.00000 & 0.00000 & 0.00000 & 0.00000 & 0.00000 & 0 \\
\hline 1.5 & -0.46264 & -0.02891 & -0.00181 & -0.00011 & -0.00001 & 0.00000 & 0.00000 & 0.00000 & 0 \\
\hline 1.6 & -1.69750 & -0.22000 & -0.02851 & -0.00370 & -0.00048 & -0.00006 & -0.00001 & 0.00000 & 0 \\
\hline 1.7 & -4.64274 & -1.11472 & -0.26764 & -0.06426 & -0.01543 & -0.00370 & -0.00089 & 0.00000 & 0 \\
\hline 1.8 & -9.53767 & -3.90663 & -1.60015 & -0.65542 & -0.26846 & -0.10996 & -0.04504 & -0.00127 & 0 \\
\hline 1.9 & -12.91556 & -8.47390 & -5.55973 & -3.64774 & -2.39328 & -1.57023 & -1.03023 & -0.19090 & 0 \\
\hline
\end{tabular}

TABLE II: Coefficient of the logarithmic term for $\Sigma_{1}$, in Feynman gauge.

\begin{tabular}{|c|c|c|c|c|c|c|c|c|c|}
\hline$M$ & $N_{s}=8$ & $N_{s}=12$ & $N_{s}=16$ & $N_{s}=20$ & $N_{s}=24$ & $N_{s}=28$ & $N_{s}=32$ & $N_{s}=48$ & $N_{s}=\infty$ \\
\hline 0.1 & 0.11387 & 0.41183 & 0.64719 & 0.80302 & 0.89547 & 0.94650 & 0.97333 & 0.99859 & 1 \\
\hline 0.2 & 0.71677 & 0.92503 & 0.98280 & 0.99634 & 0.99926 & 0.99985 & 0.99997 & 1.00000 & 1 \\
\hline 0.3 & 0.94927 & 0.99550 & 0.99965 & 0.99997 & 1.00000 & 1.00000 & 1.00000 & 1.00000 & 1 \\
\hline 0.5 & 0.99964 & 1.00000 & 1.00000 & 1.00000 & 1.00000 & 1.00000 & 1.00000 & 1.00000 & 1 \\
\hline 0.6 & 0.99999 & 1.00000 & 1.00000 & 1.00000 & 1.00000 & 1.00000 & 1.00000 & 1.00000 & 1 \\
\hline 0.7 & 1.00000 & 1.00000 & 1.00000 & 1.00000 & 1.00000 & 1.00000 & 1.00000 & 1.00000 & 1 \\
\hline 1.0 & 1.00000 & 1.00000 & 1.00000 & 1.00000 & 1.00000 & 1.00000 & 1.00000 & 1.00000 & 1 \\
\hline 1.1 & 1.00000 & 1.00000 & 1.00000 & 1.00000 & 1.00000 & 1.00000 & 1.00000 & 1.00000 & 1 \\
\hline 1.2 & 1.00000 & 1.00000 & 1.00000 & 1.00000 & 1.00000 & 1.00000 & 1.00000 & 1.00000 & 1 \\
\hline 1.3 & 1.00000 & 1.00000 & 1.00000 & 1.00000 & 1.00000 & 1.00000 & 1.00000 & 1.00000 & 1 \\
\hline 1.4 & 0.99999 & 1.00000 & 1.00000 & 1.00000 & 1.00000 & 1.00000 & 1.00000 & 1.00000 & 1 \\
\hline 1.9 & 0.11387 & 0.41183 & 0.64719 & 0.80302 & 0.89547 & 0.94650 & 0.97333 & 0.99859 & 1 \\
\hline
\end{tabular}

cases, because at one loop internal quark loops can never appear in the diagrams that enter in this as well as in the other calculations presented in this paper. These diagrams are standard and well known and can be found for example in Ref. [40].

The behavior of the tadpole diagrams as $N_{s}$ and $M$ change is particularly interesting, and we think that it is useful to include here also the values of the tadpole contributing to $\Sigma_{1}$ (although in this work we need only the results of the tadpole contributing to $\Sigma_{0}$ ). Since no pure 5-dimensional quark propagators appear in the tadpoles, for these diagrams the $\Sigma_{s t}(p)$ of Eq. (46) is diagonal in the fifth dimension and proportional to $(i \not k-4 r / a) G_{\mu \nu}(k)$. This is the same integrand of the tadpoles for Wilson fermions, and for that action in the case of the tadpole diagram contributing to $\Sigma_{1}$ it gave the result (in a general covariant gauge)

$$
T_{l}=8 \pi^{2} Z_{0}(1-1 / 4(1-\alpha))
$$

where $Z_{0}=0.154933390231 \ldots$ is a well-known integral 51]. It is then clear that for domain-wall fermions (where we have now $r=-1$ ) the behavior of the tadpole diagrams as a function of $N_{s}$ (and $M$ ) is completely determined by the damping factors in the fifth dimension. 
TABLE III: Coefficient of the logarithmic term of the vertex diagram of the vector current, in Feynman gauge.

\begin{tabular}{|c|c|c|c|c|c|c|c|c|c|}
\hline$M$ & $N_{s}=8$ & $N_{s}=12$ & $N_{s}=16$ & $N_{s}=20$ & $N_{s}=24$ & $N_{s}=28$ & $N_{s}=32$ & $N_{s}=48$ & $N_{s}=\infty$ \\
\hline 0.1 & -0.84792 & -1.02208 & -1.05864 & -1.04852 & -1.03113 & -1.01779 & -1.00952 & -1.00058 & -1 \\
\hline 0.2 & -1.04275 & -1.02123 & -1.00592 & -1.00138 & -1.00030 & -1.00006 & -1.00001 & -1.00000 & -1 \\
\hline 0.3 & -1.01354 & -1.00158 & -1.00014 & -1.00001 & -1.00000 & -1.00000 & -1.00000 & -1.00000 & -1 \\
\hline 0.4 & -1.00176 & -1.00005 & -1.00000 & -1.00000 & -1.00000 & -1.00000 & -1.00000 & -1.00000 & -1 \\
\hline 0.5 & -1.00012 & -1.00000 & -1.00000 & -1.00000 & -1.00000 & -1.00000 & -1.00000 & -1.00000 & -1 \\
\hline 0.6 & -1.00000 & -1.00000 & -1.00000 & -1.00000 & -1.00000 & -1.00000 & -1.00000 & -1.00000 & -1 \\
\hline 0.7 & -1.00000 & -1.00000 & -1.00000 & -1.00000 & -1.00000 & -1.00000 & -1.00000 & -1.00000 & -1 \\
\hline 0.8 & -1.00000 & -1.00000 & -1.00000 & -1.00000 & -1.00000 & -1.00000 & -1.00000 & -1.00000 & -1 \\
\hline 0.9 & -1.00000 & -1.00000 & -1.00000 & -1.00000 & -1.00000 & -1.00000 & -1.00000 & -1.00000 & -1 \\
\hline 1.0 & -1.00000 & -1.00000 & -1.00000 & -1.00000 & -1.00000 & -1.00000 & -1.00000 & -1.00000 & -1 \\
\hline 1.1 & -1.00000 & -1.00000 & -1.00000 & -1.00000 & -1.00000 & -1.00000 & -1.00000 & -1.00000 & -1 \\
\hline 1.2 & -1.00000 & -1.00000 & -1.00000 & -1.00000 & -1.00000 & -1.00000 & -1.00000 & -1.00000 & -1 \\
\hline 1.3 & -1.00000 & -1.00000 & -1.00000 & -1.00000 & -1.00000 & -1.00000 & -1.00000 & -1.00000 & -1 \\
\hline 1.4 & -1.00000 & -1.00000 & -1.00000 & -1.00000 & -1.00000 & -1.00000 & -1.00000 & -1.00000 & -1 \\
\hline 1.5 & -1.00012 & -1.00000 & -1.00000 & -1.00000 & -1.00000 & -1.00000 & -1.00000 & -1.00000 & -1 \\
\hline 1.6 & -1.00176 & -1.00005 & -1.00000 & -1.00000 & -1.00000 & -1.00000 & -1.00000 & -1.00000 & -1 \\
\hline 1.7 & -1.01354 & -1.00158 & -1.00014 & -1.00001 & -1.00000 & -1.00000 & -1.00000 & -1.00000 & -1 \\
\hline 1.8 & -1.04275 & -1.02123 & -1.00592 & -1.00138 & -1.00030 & -1.00006 & -1.00001 & -1.00000 & -1 \\
\hline 1.9 & -0.84792 & -1.02208 & -1.05864 & -1.04852 & -1.03113 & -1.01779 & -1.00952 & -1.00058 & -1 \\
\hline
\end{tabular}

TABLE IV: Anomalous dimension of the vector current, in Feynman gauge.

\begin{tabular}{|c|ccccccccc}
\hline \hline$M$ & $N_{s}=8$ & $N_{s}=12$ & $N_{s}=16$ & $N_{s}=20$ & $N_{s}=24$ & $N_{s}=28$ & $N_{s}=32$ & $N_{s}=48$ & $N_{s}=\infty \mid$ \\
\hline 0.1 & 0.73405 & 0.61025 & 0.41144 & 0.24550 & 0.13567 & 0.07128 & 0.03619 & 0.00199 & 0 \\
0.2 & 0.32598 & 0.09620 & 0.02312 & 0.00504 & 0.00104 & 0.00021 & 0.00004 & 0.00000 & 0 \\
0.3 & 0.06427 & 0.00608 & 0.00049 & 0.00004 & 0.00000 & 0.00000 & 0.00000 & 0.00000 \\
0.4 & 0.00733 & 0.00020 & 0.00000 & 0.00000 & 0.00000 & 0.00000 & 0.00000 & 0.00000 & 0 \\
0.5 & 0.00048 & 0.00000 & 0.00000 & 0.00000 & 0.00000 & 0.00000 & 0.00000 & 0.00000 \\
0.6 & 0.00002 & 0.00000 & 0.00000 & 0.00000 & 0.00000 & 0.00000 & 0.00000 & 0.00000 \\
0.7 & 0.00000 & 0.00000 & 0.00000 & 0.00000 & 0.00000 & 0.00000 & 0.00000 & 0.00000 & 0 \\
0.8 & 0.00000 & 0.00000 & 0.00000 & 0.00000 & 0.00000 & 0.00000 & 0.00000 & 0.00000 \\
0.9 & 0.00000 & 0.00000 & 0.00000 & 0.00000 & 0.00000 & 0.00000 & 0.00000 & 0.00000 \\
1.0 & 0.00000 & 0.00000 & 0.00000 & 0.00000 & 0.00000 & 0.00000 & 0.00000 & 0.00000 \\
1.1 & 0.00000 & 0.00000 & 0.00000 & 0.00000 & 0.00000 & 0.00000 & 0.00000 & 0.00000 \\
1.2 & 0.00000 & 0.00000 & 0.00000 & 0.00000 & 0.00000 & 0.00000 & 0.00000 & 0.00000 \\
1.3 & 0.00000 & 0.00000 & 0.00000 & 0.00000 & 0.00000 & 0.00000 & 0.00000 & 0.00000 \\
1.4 & 0.00002 & 0.00000 & 0.00000 & 0.00000 & 0.00000 & 0.00000 & 0.00000 & 0.00000 \\
1.5 & 0.00048 & 0.00000 & 0.00000 & 0.00000 & 0.00000 & 0.00000 & 0.00000 & 0.00000 \\
1.6 & 0.00733 & 0.00020 & 0.00000 & 0.00000 & 0.00000 & 0.00000 & 0.00000 & 0.00000 \\
1.7 & 0.06427 & 0.00608 & 0.00049 & 0.00004 & 0.00000 & 0.00000 & 0.00000 & 0.00000 \\
1.8 & 0.32598 & 0.09620 & 0.02312 & 0.00504 & 0.00104 & 0.00021 & 0.00004 & 0.00000 \\
1.9 & 0.73405 & 0.61025 & 0.41144 & 0.24550 & 0.13567 & 0.07128 & 0.03619 & 0.00199 \\
\hline \hline
\end{tabular}

Their general effect can be seen by looking at their leading contributions for large $N_{s}$, which enter the game in the combinations

$$
\sum_{s=1}^{N_{s}} w_{0}^{s-1} w_{0}^{N_{s}-s}=N_{s} w_{0}^{N_{s}-1}
$$

and

$$
\sum_{s=1}^{N_{s}}\left(w_{0}^{2}\right)^{s-1}=\sum_{s=1}^{N_{s}}\left(w_{0}^{2}\right)^{N_{s}-s}=\frac{1-w_{0}^{2 N_{s}}}{1-w_{0}^{2}} .
$$

These are indeed the leading expressions, in units of

$$
T_{d}=\frac{\left(1-w_{0}^{2}\right) T_{l}}{\left(1-w_{0}^{2 N_{s}}\right)^{2}},
$$

for the tadpole contributions to $\Sigma_{0}$ and $\Sigma_{1}$ respectively, in the limit of large $N_{s}$. From these expressions one can immediately see that the tadpole of $\Sigma_{0}$ vanishes when $N_{s}=\infty$, while the tadpole of $\Sigma_{1}$ gives in this limit the well-known Wilson number, $T_{l}$. The damping factors thus play a primary rôle in determining the results of the domain-wall tadpoles. We have calculated their exact expressions including all subleading terms in $N_{s}$, 
TABLE V: Anomalous dimension of the scalar density, in Feynman gauge.

\begin{tabular}{|l|lcccccccr|}
\hline \hline$M$ & $N_{s}=8$ & $N_{s}=12$ & $N_{s}=16$ & $N_{s}=20$ & $N_{s}=24$ & $N_{s}=28$ & $N_{s}=32$ & $N_{s}=48$ & $N_{s}=\infty \mid$ \\
\hline 0.1 & 3.27780 & 3.67649 & 3.58735 & 3.39105 & 3.22907 & 3.12464 & 3.06474 & 3.00372 & 3 \\
0.2 & 3.45424 & 3.15990 & 3.04087 & 3.00918 & 3.00193 & 3.00039 & 3.00008 & 3.00000 & 3 \\
0.3 & 3.10489 & 3.01080 & 3.00089 & 3.00007 & 3.00000 & 3.00000 & 3.00000 & 3.00000 & 3 \\
0.4 & 3.01260 & 3.00036 & 3.00001 & 3.00000 & 3.00000 & 3.00000 & 3.00000 & 3.00000 & 3 \\
0.5 & 3.00085 & 3.00001 & 3.00000 & 3.00000 & 3.00000 & 3.00000 & 3.00000 & 3.00000 & 3 \\
0.6 & 3.00003 & 3.00000 & 3.00000 & 3.00000 & 3.00000 & 3.00000 & 3.00000 & 3.00000 & 3 \\
0.7 & 3.00000 & 3.00000 & 3.00000 & 3.00000 & 3.00000 & 3.00000 & 3.00000 & 3.00000 & 3 \\
0.8 & 3.00000 & 3.00000 & 3.00000 & 3.00000 & 3.00000 & 3.00000 & 3.00000 & 3.00000 & 3 \\
0.9 & 3.00000 & 3.00000 & 3.00000 & 3.00000 & 3.00000 & 3.00000 & 3.00000 & 3.00000 & 3 \\
1.0 & 3.00000 & 3.00000 & 3.00000 & 3.00000 & 3.00000 & 3.00000 & 3.00000 & 3.00000 & 3 \\
1.1 & 3.00000 & 3.00000 & 3.00000 & 3.00000 & 3.00000 & 3.00000 & 3.00000 & 3.00000 & 3 \\
1.2 & 3.00000 & 3.00000 & 3.00000 & 3.00000 & 3.00000 & 3.00000 & 3.00000 & 3.00000 & 3 \\
1.3 & 3.00000 & 3.00000 & 3.00000 & 3.00000 & 3.00000 & 3.00000 & 3.00000 & 3.00000 & 3 \\
1.4 & 3.00003 & 3.00000 & 3.00000 & 3.00000 & 3.00000 & 3.00000 & 3.00000 & 3.00000 & 3 \\
1.5 & 3.00085 & 3.00001 & 3.00000 & 3.00000 & 3.00000 & 3.00000 & 3.00000 & 3.00000 & 3 \\
1.6 & 3.01260 & 3.00036 & 3.00001 & 3.00000 & 3.00000 & 3.00000 & 3.00000 & 3.00000 & 3 \\
1.7 & 3.10489 & 3.01080 & 3.00089 & 3.00007 & 3.00000 & 3.00000 & 3.00000 & 3.00000 & 3 \\
1.8 & 3.45424 & 3.15990 & 3.04087 & 3.00918 & 3.00193 & 3.00039 & 3.00008 & 3.00000 & 3 \\
1.9 & 3.27780 & 3.67649 & 3.58735 & 3.39105 & 3.22907 & 3.12464 & 3.06474 & 3.00372 & 3 \\
\hline \hline
\end{tabular}

TABLE VI: Tadpole contribution to $\Sigma_{0}$, Eq. [69), in Feynman gauge (multiplied for $16 \pi^{2}$ ).

\begin{tabular}{|r|rrrrrrrr}
\hline \hline$M$ & $N_{s}=8$ & \multicolumn{1}{c}{$N_{s}=12$} & $N_{s}=16$ & \multicolumn{1}{c}{$N_{s}=20$} & $N_{s}=24$ & $N_{s}=28$ & $N_{s}=32$ & $N_{s}=48$ \\
\hline 0.1 & 15.10361 & 16.98204 & 16.85601 & 15.31833 & 13.06260 & 10.63233 & 8.36032 & 2.59484 \\
0.2 & 18.33890 & 12.98046 & 7.73201 & 4.17637 & 2.12604 & 1.04115 & 0.49623 & 0.02182 \\
0.3 & 12.61592 & 4.97585 & 1.66803 & 0.51427 & 0.15080 & 0.04277 & 0.01184 & 0.00006 \\
0.4 & 6.03146 & 1.23559 & 0.21903 & 0.03602 & 0.00566 & 0.00086 & 0.00013 & 0.00000 \\
0.5 & 2.10263 & 0.20309 & 0.01717 & 0.00135 & 0.00010 & 0.00001 & 0.00000 & 0.00000 \\
0.6 & 0.51309 & 0.02003 & 0.00069 & 0.00002 & 0.00000 & 0.00000 & 0.00000 & 0.00000 \\
0.7 & 0.07598 & 0.00093 & 0.00001 & 0.00000 & 0.00000 & 0.00000 & 0.00000 & 0.00000 \\
0.8 & 0.00476 & 0.00001 & 0.00000 & 0.00000 & 0.00000 & 0.00000 & 0.00000 & 0.00000 \\
0.9 & 0.00004 & 0.00000 & 0.00000 & 0.00000 & 0.00000 & 0.00000 & 0.00000 & 0.00000 \\
1.0 & 0.00000 & 0.00000 & 0.00000 & 0.00000 & 0.00000 & 0.00000 & 0.00000 & 0.00000 \\
1.1 & -0.00004 & -0.00000 & -0.00000 & -0.00000 & -0.00000 & -0.00000 & -0.00000 & -0.00000 \\
1.2 & -0.00476 & -0.00001 & -0.00000 & -0.00000 & -0.00000 & -0.00000 & -0.00000 & -0.00000 \\
1.3 & -0.07598 & -0.00093 & -0.00001 & -0.00000 & -0.00000 & -0.00000 & -0.00000 & -0.00000 \\
1.4 & -0.51309 & -0.02003 & -0.00069 & -0.00002 & -0.00000 & -0.00000 & -0.00000 & -0.00000 \\
1.5 & -2.10263 & -0.20309 & -0.01717 & -0.00135 & -0.00010 & -0.00001 & -0.00000 & -0.00000 \\
1.6 & -6.03146 & -1.23559 & -0.21903 & -0.03602 & -0.00566 & -0.00086 & -0.00013 & -0.00000 \\
1.7 & -12.61592 & -4.97585 & -1.66803 & -0.51427 & -0.15080 & -0.04277 & -0.01184 & -0.00006 \\
1.8 & -18.33890 & -12.98046 & -7.73201 & -4.17637 & -2.12604 & -1.04115 & -0.49623 & -0.02182 \\
1.9 & -15.10361 & -16.98204 & -16.85601 & -15.31833 & -13.06260 & -10.63233 & -8.36032 & -2.59484 \\
\hline \hline
\end{tabular}

and the tadpole contribution to $\Sigma_{0}$ turns out to be equal to

$$
4 T_{d}\left[N_{s}\left(1+w_{0}^{2\left(N_{s}+1\right)}\right) w_{0}^{N_{s}-1}-2 w_{0}^{N_{s}+1} \frac{1-w_{0}^{2 N_{s}}}{1-w_{0}^{2}}\right]
$$

while the tadpole contribution to $\Sigma_{1}$ turns out to be equal to

$$
T_{d}\left[\left(1+w_{0}^{2\left(N_{s}+1\right)}\right) \frac{1-w_{0}^{2 N_{s}}}{1-w_{0}^{2}}-2 N_{s} w_{0}^{2 N_{s}}\right] .
$$

Numerical values of these tadpoles for various choices of $N_{s}$ and $M$ are collected in Tables VI and VII where, as in the rest of the paper, we also show the corresponding values for the limiting case of infinite extent in the fifth dimension. In the case of $\Sigma_{0}$ we observe that its tadpole contribution presents wide variations with $N_{s}$ and $M$, so that in some cases it turns out to be small while in other cases it can be substantially large. The tadpole contributing to $\Sigma_{1}$ instead has smaller variations. This suggests that some care should be used when talking about tadpole dominance in relation to domain-wall fermions. In fact, the tadpoles contributing to $\Sigma_{0}$ and $\Sigma_{1}$ even decrease toward zero when $M \rightarrow 0$ or $M \rightarrow 2$, as we will see in Sect. VIII 
TABLE VII: Tadpole contribution to $\Sigma_{1}$, Eq. (70), in Feynman gauge (multiplied for $16 \pi^{2}$ ).

\begin{tabular}{|c|c|c|c|c|c|c|c|c|c|}
\hline$M$ & $N_{s}=8$ & $N_{s}=12$ & $N_{s}=16$ & $N_{s}=20$ & $N_{s}=24$ & $N_{s}=28$ & $N_{s}=32$ & $N_{s}=48$ & $N_{s}=\infty$ \\
\hline 0.1 & 6.88684 & 8.89791 & 10.28165 & 11.14950 & 11.65586 & 11.93540 & 12.08339 & 12.22491 & 12.23305 \\
\hline 0.2 & 10.71422 & 11.82437 & 12.13713 & 12.21230 & 12.22878 & 12.23220 & 12.23289 & 12.23305 & 12.23305 \\
\hline 0.3 & 11.95987 & 12.20785 & 12.23105 & 12.23290 & 12.23304 & 12.23305 & 12.23305 & 12.23305 & 12.23305 \\
\hline 0.4 & 12.20239 & 12.23224 & 12.23303 & 12.23305 & 12.23305 & 12.23305 & 12.23305 & 12.23305 & 12.23305 \\
\hline 0.5 & 12.23104 & 12.23304 & 12.23305 & 12.23305 & 12.23305 & 12.23305 & 12.23305 & 12.23305 & 12.23305 \\
\hline 0.6 & 12.23299 & 12.23305 & 12.23305 & 12.23305 & 12.23305 & 12.23305 & 12.23305 & 12.23305 & 12.23305 \\
\hline 0.7 & 12.23305 & 12.23305 & 12.23305 & 12.23305 & 12.23305 & 12.23305 & 12.23305 & 12.23305 & 12.23305 \\
\hline 0.8 & 12.23305 & 12.23305 & 12.23305 & 12.23305 & 12.23305 & 12.23305 & 12.23305 & 12.23305 & 12.23305 \\
\hline 0.9 & 12.23305 & 12.23305 & 12.23305 & 12.23305 & 12.23305 & 12.23305 & 12.23305 & 12.23305 & 12.23305 \\
\hline 1.0 & 12.23305 & 12.23305 & 12.23305 & 12.23305 & 12.23305 & 12.23305 & 12.23305 & 12.23305 & 12.23305 \\
\hline 1.1 & 12.23305 & 12.23305 & 12.23305 & 12.23305 & 12.23305 & 12.23305 & 12.23305 & 12.23305 & 12.23305 \\
\hline 1.2 & 12.23305 & 12.23305 & 12.23305 & 12.23305 & 12.23305 & 12.23305 & 12.23305 & 12.23305 & 12.23305 \\
\hline 1.3 & 12.23305 & 12.23305 & 12.23305 & 12.23305 & 12.23305 & 12.23305 & 12.23305 & 12.23305 & 12.23305 \\
\hline 1.4 & 12.23299 & 12.23305 & 12.23305 & 12.23305 & 12.23305 & 12.23305 & 12.23305 & 12.23305 & 12.23305 \\
\hline 1.5 & 12.23104 & 12.23304 & 12.23305 & 12.23305 & 12.23305 & 12.23305 & 12.23305 & 12.23305 & 12.23305 \\
\hline 1.6 & 12.20239 & 12.23224 & 12.23303 & 12.23305 & 12.23305 & 12.23305 & 12.23305 & 12.23305 & 12.23305 \\
\hline 1.7 & 11.95987 & 12.20785 & 12.23105 & 12.23290 & 12.23304 & 12.23305 & 12.23305 & 12.23305 & 12.23305 \\
\hline 1.8 & 10.71422 & 11.82437 & 12.13713 & 12.21230 & 12.22878 & 12.23220 & 12.23289 & 12.23305 & 12.23305 \\
\hline 1.9 & 6.88684 & 8.89791 & 10.28165 & 11.14950 & 11.65586 & 11.93540 & 12.08339 & 12.22491 & 12.23305 \\
\hline
\end{tabular}

TABLE VIII: Coefficient of $\bar{g}^{2}$ for the contribution of the half-circle diagram to $\Sigma_{0}$, in Feynman gauge.

\begin{tabular}{|l|ccccccccc}
\hline \hline$M$ & $N_{s}=8$ & $N_{s}=12$ & $N_{s}=16$ & $N_{s}=20$ & $N_{s}=24$ & $N_{s}=28$ & $N_{s}=32$ & $N_{s}=48$ & $N_{s}=\infty$ \\
\hline 0.1 & 2.75558 & 2.69676 & 2.39676 & 2.00277 & 1.60286 & 1.24317 & 0.94186 & 0.26825 & 0 \\
0.2 & 3.48374 & 2.22615 & 1.24197 & 0.64382 & 0.31901 & 0.15331 & 0.07208 & 0.00306 \\
0.3 & 2.67348 & 1.01173 & 0.33194 & 0.10111 & 0.02942 & 0.00830 & 0.00227 & 0.00001 \\
0.4 & 1.52701 & 0.31852 & 0.05710 & 0.00946 & 0.00147 & 0.00022 & 0.00003 & 0.00000 & 0 \\
0.5 & 0.68927 & 0.07235 & 0.00641 & 0.00051 & 0.00004 & 0.00000 & 0.00000 & 0.00000 \\
0.6 & 0.24943 & 0.01188 & 0.00046 & 0.00002 & 0.00000 & 0.00000 & 0.00000 & 0.00000 \\
0.7 & 0.07417 & 0.00157 & 0.00003 & 0.00000 & 0.00000 & 0.00000 & 0.00000 & 0.00000 & 0 \\
0.8 & 0.02085 & 0.00029 & 0.00001 & 0.00000 & 0.00000 & 0.00000 & 0.00000 & 0.00000 \\
0.9 & 0.00770 & 0.00012 & 0.00000 & 0.00000 & 0.00000 & 0.00000 & 0.00000 & 0.00000 & 0 \\
1.0 & 0.00418 & 0.00008 & 0.00000 & 0.00000 & 0.00000 & 0.00000 & 0.00000 & 0.00000 \\
1.1 & 0.00286 & 0.00007 & 0.00000 & 0.00000 & 0.00000 & 0.00000 & 0.00000 & 0.00000 \\
1.2 & 0.00304 & 0.00007 & 0.00000 & 0.00000 & 0.00000 & 0.00000 & 0.00000 & 0.00000 \\
1.3 & 0.01346 & 0.00021 & 0.00001 & 0.00000 & 0.00000 & 0.00000 & 0.00000 & 0.00000 \\
1.4 & 0.06983 & 0.00270 & 0.00009 & 0.00000 & 0.00000 & 0.00000 & 0.00000 & 0.00000 \\
1.5 & 0.24400 & 0.02258 & 0.00186 & 0.00013 & 0.00001 & 0.00000 & 0.00000 & 0.00000 \\
1.6 & 0.60517 & 0.11252 & 0.01902 & 0.00304 & 0.00045 & 0.00006 & 0.00001 & 0.00000 \\
1.7 & 1.12385 & 0.36321 & 0.10935 & 0.03157 & 0.00886 & 0.00243 & 0.00064 & 0.00000 \\
1.8 & 1.56704 & 0.77955 & 0.36471 & 0.16644 & 0.07499 & 0.03349 & 0.01486 & 0.00053 \\
1.9 & 1.40741 & 1.01047 & 0.66666 & 0.41011 & 0.23700 & 0.12837 & 0.06403 & -0.00472 \\
\hline \hline
\end{tabular}

Given the strong dependence on $M$ and $N_{s}$, tadpole improvement (at least in its more common form) seems in general not to be an appropriate tool with regard to the residual mass, which is here associated with the additive mass renormalization arising from the one-loop selfenergy, Eq. (48). The contribution of the one-loop diagrams to this mass can be inferred from the numbers for $\Sigma_{0}$ presented in Tables $[\mathrm{X}$ and $\mathrm{XI}$ (in Feynman and Landau gauge respectively), which take into account the results for the half-circle diagram given in Tables VIII and IX Since we have performed the calculations in a general covariant gauge, we can express all the quantities presented in this paper in the form

$$
A+(1-\alpha) B
$$

where $A$ and $A+B$ provide the answer in Feynman and Landau gauge respectively, and $B$ is a number which remains the same when using fermion formulations rather diverse like domain-wall with an infinite extent of the fifth dimension, Wilson or overlap. The values of $B$ in the case of the contribution of the half-circle diagram to $\Sigma_{0}$ at finite $N_{s}$ are given in Table IX If we now compare the numbers shown in Tables $\mathrm{X}$ and $\mathrm{XI}$, we can deduce that, even after the result for the tadpole is included, the 
TABLE IX: Coefficient of $\bar{g}^{2}$ for the part proportional to $1-\alpha$ of the contribution of the half-circle diagram to $\Sigma_{0}$.

\begin{tabular}{|c|c|c|c|c|c|c|c|c|c|}
\hline$M$ & $N_{s}=8$ & $N_{s}=12$ & $N_{s}=16$ & $N_{s}=20$ & $N_{s}=24$ & $N_{s}=28$ & $N_{s}=32$ & $N_{s}=48$ & $N_{s}=\infty$ \\
\hline 0.1 & 3.19742 & 3.77191 & 3.84954 & 3.56027 & 3.07177 & 2.52079 & 1.99387 & 0.62668 & 0 \\
\hline 0.2 & 4.00781 & 2.95012 & 1.79210 & 0.97875 & 0.50169 & 0.24683 & 0.11804 & 0.00523 & 0 \\
\hline 0.3 & 2.81125 & 1.14035 & 0.38726 & 0.12026 & 0.03543 & 0.01008 & 0.00280 & 0.00001 & 0 \\
\hline 0.4 & 1.35920 & 0.28390 & 0.05078 & 0.00839 & 0.00133 & 0.00020 & 0.00003 & 0.00000 & 0 \\
\hline 0.5 & 0.47745 & 0.04674 & 0.00398 & 0.00032 & 0.00002 & 0.00000 & 0.00000 & 0.00000 & 0 \\
\hline 0.6 & 0.11737 & 0.00463 & 0.00016 & 0.00001 & 0.00000 & 0.00000 & 0.00000 & 0.00000 & 0 \\
\hline 0.7 & 0.01755 & 0.00022 & 0.00000 & 0.00000 & 0.00000 & 0.00000 & 0.00000 & 0.00000 & 0 \\
\hline 0.8 & 0.00112 & 0.00000 & 0.00000 & 0.00000 & 0.00000 & 0.00000 & 0.00000 & 0.00000 & 0 \\
\hline 0.9 & 0.00001 & 0.00000 & 0.00000 & 0.00000 & 0.00000 & 0.00000 & 0.00000 & 0.00000 & 0 \\
\hline 1.0 & 0.00000 & 0.00000 & 0.00000 & 0.00000 & 0.00000 & 0.00000 & 0.00000 & 0.00000 & 0 \\
\hline 1.1 & -0.00001 & 0.00000 & 0.00000 & 0.00000 & 0.00000 & 0.00000 & 0.00000 & 0.00000 & 0 \\
\hline 1.2 & -0.00126 & 0.00000 & 0.00000 & 0.00000 & 0.00000 & 0.00000 & 0.00000 & 0.00000 & 0 \\
\hline 1.3 & -0.02044 & -0.00025 & 0.00000 & 0.00000 & 0.00000 & 0.00000 & 0.00000 & 0.00000 & 0 \\
\hline 1.4 & -0.13917 & -0.00539 & -0.00018 & -0.00001 & 0.00000 & 0.00000 & 0.00000 & 0.00000 & 0 \\
\hline 1.5 & -0.57387 & -0.05480 & -0.00461 & -0.00036 & -0.00003 & 0.00000 & 0.00000 & 0.00000 & 0 \\
\hline 1.6 & -1.65669 & -0.33389 & -0.05873 & -0.00961 & -0.00150 & -0.00023 & -0.00003 & 0.00000 & 0 \\
\hline 1.7 & -3.49885 & -1.34763 & -0.44675 & -0.13687 & -0.03997 & -0.01130 & -0.00312 & -0.00002 & 0 \\
\hline 1.8 & -5.17527 & -3.54181 & -2.07409 & -1.10946 & -0.56133 & -0.27375 & -0.13008 & -0.00568 & 0 \\
\hline 1.9 & -4.39070 & -4.73341 & -4.58402 & -4.10098 & -3.46030 & -2.79565 & -2.18639 & -0.67074 & 0 \\
\hline
\end{tabular}

TABLE X: Coefficient of $\bar{g}^{2}$ for the complete result of $\Sigma_{0}$, in Feynman gauge.

\begin{tabular}{|c|c|c|c|c|c|c|c|c|c|}
\hline $\bar{M}$ & $N_{s}=8$ & $N_{s}=12$ & $N_{s}=16$ & $N_{s}=20$ & $N_{s}=24$ & $N_{s}=28$ & $N_{s}=32$ & $N_{s}=48$ & $N_{s}=\infty$ \\
\hline 0.1 & 17.85919 & 19.67880 & 19.25277 & 17.32109 & 14.66546 & 11.87550 & 9.30218 & 2.86309 & 0 \\
\hline 0.2 & 21.82264 & 15.20660 & 8.97397 & 4.82019 & 2.44504 & 1.19446 & 0.56831 & 0.02488 & 0 \\
\hline 0.3 & 15.28940 & 5.98758 & 1.99997 & 0.61538 & 0.18022 & 0.05106 & 0.01412 & 0.00007 & 0 \\
\hline 0.5 & 2.79190 & 0.27543 & 0.02358 & 0.00186 & 0.00014 & 0.00001 & 0.00000 & 0.00000 & 0 \\
\hline 0.6 & 0.76252 & 0.03191 & 0.00115 & 0.00004 & 0.00000 & 0.00000 & 0.00000 & 0.00000 & 0 \\
\hline 0.7 & 0.15015 & 0.00250 & 0.00004 & 0.00000 & 0.00000 & 0.00000 & 0.00000 & 0.00000 & 0 \\
\hline 0.9 & 0.00774 & 0.00012 & 0.00000 & 0.00000 & 0.00000 & 0.00000 & 0.00000 & 0.00000 & 0 \\
\hline 1.0 & 0.00418 & 0.00008 & 0.00000 & 0.00000 & 0.00000 & 0.00000 & 0.00000 & 0.00000 & 0 \\
\hline 1.1 & 0.00283 & 0.00007 & 0.00000 & 0.00000 & 0.00000 & 0.00000 & 0.00000 & 0.00000 & 0 \\
\hline 1.2 & -0.00172 & 0.00006 & 0.00000 & 0.00000 & 0.00000 & 0.00000 & 0.00000 & 0.00000 & 0 \\
\hline 1.3 & -0.06252 & -0.00073 & 0.00000 & 0.00000 & 0.00000 & 0.00000 & 0.00000 & 0.00000 & 0 \\
\hline 1.4 & -0.44327 & -0.01733 & -0.00060 & -0.00002 & 0.00000 & 0.00000 & 0.00000 & 0.00000 & 0 \\
\hline 1.9 & -13.69620 & -15.97157 & -16.18935 & -14.90821 & -12.82561 & -10.50396 & -8.29629 & -2.59956 & 0 \\
\hline
\end{tabular}

contribution to $\Sigma_{0}$ proportional to $1-\alpha$ is in general not equal to zero, which is what one would have instead obtained if $N_{s}=\infty$ or if Wilson or overlap fermions were used. All this means that at finite $N_{s}$ the residual mass, which is derived from $\Sigma_{0}$ according to Eq. (48), is not a gauge invariant quantity anymore. Although numerically the deviations from gauge invariance remain in most cases rather small (because there are large cancellations between the contributions of the half-circle and tadpole diagrams), we encounter here another of the pathological features of the domain-wall theory truncated at finite $N_{s}$. Thus, anomalous dimensions as well as terms pro- portional to $1-\alpha$, two of the quantities that remain the same when using a wide variety of fermionic actions, assume instead new values when the theory of domain-wall fermions is truncated at a finite $N_{s}$.

It is also interesting to compare the one-loop results presented here for $\Sigma_{0}$ to the numbers that one obtains using Wilson fermions, which are -51.43471 for the unimproved and -31.98644 for the improved (with $c_{s w}=1$ ) case. We can then see that the domain-wall results are much smaller than the Wilson numbers, even for $N_{s}$ as small as 8 .

The values of $a m_{r e s}^{(1)}$ which come out from our results 
TABLE XI: Coefficient of $\bar{g}^{2}$ for the complete result of $\Sigma_{0}$, in Landau gauge.

\begin{tabular}{|c|c|c|c|c|c|c|c|c|c|}
\hline$M$ & $N_{s}=8$ & $N_{s}=12$ & $N_{s}=16$ & $N_{s}=20$ & $N_{s}=24$ & $N_{s}=28$ & $N_{s}=32$ & $N_{s}=48$ & $N_{s}=\infty$ \\
\hline 0.1 & 17.28070 & 19.20520 & 18.88831 & 17.05178 & 14.47158 & 11.73820 & 9.20597 & 2.84106 & 0 \\
\hline 0.2 & 21.24572 & 14.91161 & 8.83308 & 4.75484 & 2.41522 & 1.18100 & 0.56229 & 0.02466 & 0 \\
\hline 0.3 & 14.94668 & 5.88397 & 1.97022 & 0.60708 & 0.17795 & 0.05045 & 0.01396 & 0.00007 & 0 \\
\hline 0.4 & 7.40981 & 1.52910 & 0.27215 & 0.04487 & 0.00704 & 0.00107 & 0.00016 & 0.00000 & 0 \\
\hline 0.5 & 2.74369 & 0.27141 & 0.02327 & 0.00184 & 0.00014 & 0.00001 & 0.00000 & 0.00000 & 0 \\
\hline 0.6 & 0.75162 & 0.03153 & 0.00114 & 0.00004 & 0.00000 & 0.00000 & 0.00000 & 0.00000 & 0 \\
\hline 0.7 & 0.14871 & 0.00249 & 0.00004 & 0.00000 & 0.00000 & 0.00000 & 0.00000 & 0.00000 & 0 \\
\hline 0.8 & 0.02554 & 0.00031 & 0.00001 & 0.00000 & 0.00000 & 0.00000 & 0.00000 & 0.00000 & 0 \\
\hline 0.9 & 0.00774 & 0.00012 & 0.00000 & 0.00000 & 0.00000 & 0.00000 & 0.00000 & 0.00000 & 0 \\
\hline 1.0 & 0.00418 & 0.00008 & 0.00000 & 0.00000 & 0.00000 & 0.00000 & 0.00000 & 0.00000 & 0 \\
\hline 1.1 & 0.00283 & 0.00007 & 0.00000 & 0.00000 & 0.00000 & 0.00000 & 0.00000 & 0.00000 & 0 \\
\hline 1.2 & -0.00179 & 0.00006 & 0.00000 & 0.00000 & 0.00000 & 0.00000 & 0.00000 & 0.00000 & 0 \\
\hline 1.3 & -0.06397 & -0.00074 & 0.00000 & 0.00000 & 0.00000 & 0.00000 & 0.00000 & 0.00000 & 0 \\
\hline 1.4 & -0.45417 & -0.01771 & -0.00061 & -0.00002 & 0.00000 & 0.00000 & 0.00000 & 0.00000 & 0 \\
\hline 1.5 & -1.90684 & -0.18453 & -0.01562 & -0.00124 & -0.00009 & -0.00001 & 0.00000 & 0.00000 & 0 \\
\hline 1.6 & -5.57512 & -1.14806 & -0.20399 & -0.03359 & -0.00530 & -0.00081 & -0.00012 & 0.00000 & 0 \\
\hline 1.7 & -11.83694 & -4.71631 & -1.58842 & -0.49100 & -0.14421 & -0.04095 & -0.01136 & -0.00006 & 0 \\
\hline 1.8 & -17.36240 & -12.49760 & -7.50839 & -4.07530 & -2.08087 & -1.02111 & -0.48740 & -0.02151 & 0 \\
\hline 1.9 & -14.31100 & -16.45947 & -16.55937 & -15.17962 & -13.02025 & -10.64153 & -8.39260 & -2.62158 & 0 \\
\hline
\end{tabular}

TABLE XII: Residual mass in lattice units at $\beta=6$ in Feynman gauge.

\begin{tabular}{|c|c|c|c|c|c|c|c|c|c|}
\hline $\bar{M}$ & $N_{s}=8$ & $N_{s}=12$ & $N_{s}=16$ & $N_{s}=20$ & $N_{s}=24$ & $N_{s}=28$ & $N_{s}=32$ & $N_{s}=48$ & $N_{s}=\infty$ \\
\hline 0.2 & -0.24466 & -0.15313 & -0.08590 & -0.04485 & -0.02234 & -0.01078 & -0.00508 & -0.00022 & 0 \\
\hline 0.4 & -0.07457 & -0.01452 & -0.00251 & -0.00041 & -0.00006 & -0.00001 & 0.00000 & 0.00000 & 0 \\
\hline 0.5 & -0.02650 & -0.00251 & -0.00021 & -0.00002 & 0.00000 & 0.00000 & 0.00000 & 0.00000 & 0 \\
\hline 0.6 & -0.00699 & -0.00028 & -0.00001 & 0.00000 & 0.00000 & 0.00000 & 0.00000 & 0.00000 & 0 \\
\hline 0.9 & -0.00007 & 0.00000 & 0.00000 & 0.00000 & 0.00000 & 0.00000 & 0.00000 & 0.00000 & 0 \\
\hline 1.0 & -0.00004 & 0.00000 & 0.00000 & 0.00000 & 0.00000 & 0.00000 & 0.00000 & 0.00000 & 0 \\
\hline 1.1 & -0.00002 & 0.00000 & 0.00000 & 0.00000 & 0.00000 & 0.00000 & 0.00000 & 0.00000 & 0 \\
\hline 1.2 & 0.00001 & 0.00000 & 0.00000 & 0.00000 & 0.00000 & 0.00000 & 0.00000 & 0.00000 & 0 \\
\hline 1.3 & 0.00047 & 0.00001 & 0.00000 & 0.00000 & 0.00000 & 0.00000 & 0.00000 & 0.00000 & 0 \\
\hline 1.4 & 0.00319 & 0.00013 & 0.00000 & 0.00000 & 0.00000 & 0.00000 & 0.00000 & 0.00000 & 0 \\
\hline 1.9 & 0.03385 & 0.08119 & 0.10149 & 0.10278 & 0.09314 & 0.07875 & 0.06353 & 0.02074 & 0 \\
\hline
\end{tabular}

for $\Sigma_{0}$ are reported in Tables XII to XV One can immediately notice that the deviations from the case of exact chiral symmetry are much more pronounced when $M$ is close to 0.1 or 1.9 . Since $M=1.8$ is at present a preferred choice for Monte Carlo simulations, as it appears to minimize chiral violations at the nonperturbative level 9, 10], we can focus on this value of $M$ and observe that at one loop the critical mass seems to be still large up to $N_{s}=16$ or $N_{s}=20$, becoming then somewhat smaller for $N_{s}=24$ and higher. Since the tree level and one-loop contributions are of a different sign, it could also be that the two-loop expression enters again with a negative sign and that its effect is to damp the chiral violations that we have obtained. But if higher-order corrections do not at the end strongly compensate the results of the residual mass at one loop, all this suggests that values like $N_{s}=24$ would be better choices for the simulations.

The region around $M=1.8$ suffers perturbatively rather badly from another problem as well. When $M$ is indeed close to 0.1 or 1.9 , the numerical convergence of the integrals (which are computationally quite demanding, being in this case sums over a six-dimensional space) is much worse then when $M$ is nearer to 1 . This behavior had already been noticed in the calculations for the 
TABLE XIII: Residual mass in lattice units at $\beta=6$ in Landau gauge.

\begin{tabular}{|c|c|c|c|c|c|c|c|c|c|}
\hline $\bar{M}$ & $N_{s}=8$ & $N_{s}=12$ & $N_{s}=16$ & $N_{s}=20$ & $N_{s}=24$ & $N_{s}=28$ & $N_{s}=32$ & $N_{s}=48$ & $N_{s}=\infty$ \\
\hline 0.1 & -0.22770 & -0.21582 & -0.19469 & -0.16708 & -0.13735 & -0.10905 & -0.08425 & -0.02520 & 0 \\
\hline 0.2 & -0.23978 & -0.15064 & -0.08471 & -0.04430 & -0.02209 & -0.01067 & -0.00503 & -0.00022 & 0 \\
\hline 0.3 & -0.15560 & -0.05674 & -0.01833 & -0.00553 & -0.00160 & -0.00045 & -0.00012 & 0.00000 & 0 \\
\hline 0.4 & -0.07331 & -0.01430 & -0.00248 & -0.00040 & -0.00006 & -0.00001 & 0.00000 & 0.00000 & 0 \\
\hline 0.5 & -0.02610 & -0.00247 & -0.00021 & -0.00002 & 0.00000 & 0.00000 & 0.00000 & 0.00000 & 0 \\
\hline 0.6 & -0.00690 & -0.00028 & -0.00001 & 0.00000 & 0.00000 & 0.00000 & 0.00000 & 0.00000 & 0 \\
\hline 0.7 & -0.00132 & -0.00002 & 0.00000 & 0.00000 & 0.00000 & 0.00000 & 0.00000 & 0.00000 & 0 \\
\hline 0.8 & -0.00022 & 0.00000 & 0.00000 & 0.00000 & 0.00000 & 0.00000 & 0.00000 & 0.00000 & 0 \\
\hline 0.9 & -0.00007 & 0.00000 & 0.00000 & 0.00000 & 0.00000 & 0.00000 & 0.00000 & 0.00000 & 0 \\
\hline 1.0 & -0.00004 & 0.00000 & 0.00000 & 0.00000 & 0.00000 & 0.00000 & 0.00000 & 0.00000 & 0 \\
\hline 1.1 & -0.00002 & 0.00000 & 0.00000 & 0.00000 & 0.00000 & 0.00000 & 0.00000 & 0.00000 & 0 \\
\hline 1.2 & 0.00001 & 0.00000 & 0.00000 & 0.00000 & 0.00000 & 0.00000 & 0.00000 & 0.00000 & 0 \\
\hline 1.3 & 0.00048 & 0.00001 & 0.00000 & 0.00000 & 0.00000 & 0.00000 & 0.00000 & 0.00000 & 0 \\
\hline 1.4 & 0.00328 & 0.00014 & 0.00000 & 0.00000 & 0.00000 & 0.00000 & 0.00000 & 0.00000 & 0 \\
\hline 1.5 & 0.01317 & 0.00137 & 0.00012 & 0.00001 & 0.00000 & 0.00000 & 0.00000 & 0.00000 & 0 \\
\hline 1.6 & 0.03632 & 0.00830 & 0.00154 & 0.00026 & 0.00004 & 0.00001 & 0.00000 & 0.00000 & 0 \\
\hline 1.7 & 0.07054 & 0.03276 & 0.01172 & 0.00374 & 0.00112 & 0.00032 & 0.00009 & 0.00000 & 0 \\
\hline 1.8 & 0.08620 & 0.08078 & 0.05326 & 0.03026 & 0.01587 & 0.00793 & 0.00383 & 0.00017 & 0 \\
\hline 1.9 & 0.03905 & 0.08531 & 0.10461 & 0.10507 & 0.09478 & 0.07991 & 0.06434 & 0.02093 & 0 \\
\hline
\end{tabular}

TABLE XIV: Residual mass in lattice units at $\beta=5.2$ in Feynman gauge.

\begin{tabular}{|c|c|c|c|c|c|c|c|c|c|}
\hline$M$ & $N_{s}=8$ & $N_{s}=12$ & $N_{s}=16$ & $N_{s}=20$ & $N_{s}=24$ & $N_{s}=28$ & $N_{s}=32$ & $N_{s}=48$ & $N_{s}=\infty$ \\
\hline 0.1 & -0.25578 & -0.24538 & -0.22278 & -0.19185 & -0.15803 & -0.12564 & -0.09715 & -0.02910 & 0 \\
\hline 0.2 & -0.27300 & -0.17289 & -0.09756 & -0.05111 & -0.02552 & -0.01233 & -0.00582 & -0.00025 & 0 \\
\hline 0.4 & -0.08439 & -0.01653 & -0.00287 & -0.00047 & -0.00007 & -0.00001 & 0.00000 & 0.00000 & 0 \\
\hline 0.5 & -0.03013 & -0.00287 & -0.00024 & -0.00002 & 0.00000 & 0.00000 & 0.00000 & 0.00000 & 0 \\
\hline 0.6 & -0.00798 & -0.00033 & -0.00001 & 0.00000 & 0.00000 & 0.00000 & 0.00000 & 0.00000 & 0 \\
\hline 0.9 & -0.00008 & 0.00000 & 0.00000 & 0.00000 & 0.00000 & 0.00000 & 0.00000 & 0.00000 & 0 \\
\hline 1.0 & -0.00004 & 0.00000 & 0.00000 & 0.00000 & 0.00000 & 0.00000 & 0.00000 & 0.00000 & 0 \\
\hline 1.1 & -0.00003 & 0.00000 & 0.00000 & 0.00000 & 0.00000 & 0.00000 & 0.00000 & 0.00000 & 0 \\
\hline 1.2 & 0.00001 & 0.00000 & 0.00000 & 0.00000 & 0.00000 & 0.00000 & 0.00000 & 0.00000 & 0 \\
\hline 1.3 & 0.00055 & 0.00001 & 0.00000 & 0.00000 & 0.00000 & 0.00000 & 0.00000 & 0.00000 & 0 \\
\hline 1.4 & 0.00377 & 0.00015 & 0.00001 & 0.00000 & 0.00000 & 0.00000 & 0.00000 & 0.00000 & 0 \\
\hline 1.9 & 0.05165 & 0.10194 & 0.12252 & 0.12214 & 0.10980 & 0.09239 & 0.07430 & 0.02412 & 0 \\
\hline
\end{tabular}

$N_{s}=\infty$ limit presented in [40], however now the convergence is even slower than in that case, and it also tends to become worse when $N_{s}$ is small.

The values of the residual mass $a m_{r e s}^{(1)}$, that is after the one-loop corrections are added to the tree-level expression, have the same sign of the critical mass of Wilson fermions only for $M \gtrsim 1.2$. In this region of $M$ they are positive, at least for even $N_{s}$ and if the coupling is not very small. If one looks at the columns corresponding to $N_{s}=8$, our results seem to indicate that the minimal amount of chiral violations is attained for $M \sim 1.2$ (in Feynman or Landau gauge). The optimal choice of $M$ from the point of view of 1-loop calculations would then be around these values. This effect can be related to the renormalization of $M$, a quantity which is not protected by chiral symmetry and is moved by radiative corrections away from its free value, $M=1$ (where the tree-level residual mass vanishes). One can conjecture that higherloop corrections and nonperturbative effects would shift this optimal value further on, until the chiral violations approach a minimal point around $M=1.8$, which seems to be the choice that provides the smallest residual mass in Monte Carlo simulations. It could be that the results of the one-loop calculations for $M=1.8$ are not substantially changed once higher-loop corrections are included, 
TABLE XV: Residual mass in lattice units at $\beta=5.2$ in Landau gauge.

\begin{tabular}{|c|c|c|c|c|c|c|c|c|c|}
\hline$M$ & $N_{s}=8$ & $N_{s}=12$ & $N_{s}=16$ & $N_{s}=20$ & $N_{s}=24$ & $N_{s}=28$ & $N_{s}=32$ & $N_{s}=48$ & $N_{s}=\infty$ \\
\hline 0.1 & -0.25014 & -0.24077 & -0.21923 & -0.18923 & -0.15614 & -0.12430 & -0.09621 & -0.02889 & 0 \\
\hline 0.2 & -0.26738 & -0.17001 & -0.09619 & -0.05047 & -0.02523 & -0.01220 & -0.00576 & -0.00025 & 0 \\
\hline 0.3 & -0.17502 & -0.06438 & -0.02089 & -0.00632 & -0.00183 & -0.00051 & -0.00014 & 0.00000 & 0 \\
\hline 0.4 & -0.08294 & -0.01629 & -0.00283 & -0.00046 & -0.00007 & -0.00001 & 0.00000 & 0.00000 & 0 \\
\hline 0.5 & -0.02966 & -0.00283 & -0.00024 & -0.00002 & 0.00000 & 0.00000 & 0.00000 & 0.00000 & 0 \\
\hline 0.6 & -0.00787 & -0.00032 & -0.00001 & 0.00000 & 0.00000 & 0.00000 & 0.00000 & 0.00000 & 0 \\
\hline 0.7 & -0.00151 & -0.00002 & 0.00000 & 0.00000 & 0.00000 & 0.00000 & 0.00000 & 0.00000 & 0 \\
\hline 0.8 & -0.00025 & 0.00000 & 0.00000 & 0.00000 & 0.00000 & 0.00000 & 0.00000 & 0.00000 & 0 \\
\hline 0.9 & -0.00008 & 0.00000 & 0.00000 & 0.00000 & 0.00000 & 0.00000 & 0.00000 & 0.00000 & 0 \\
\hline 1.0 & -0.00004 & 0.00000 & 0.00000 & 0.00000 & 0.00000 & 0.00000 & 0.00000 & 0.00000 & 0 \\
\hline 1.1 & -0.00003 & 0.00000 & 0.00000 & 0.00000 & 0.00000 & 0.00000 & 0.00000 & 0.00000 & 0 \\
\hline 1.2 & 0.00002 & 0.00000 & 0.00000 & 0.00000 & 0.00000 & 0.00000 & 0.00000 & 0.00000 & 0 \\
\hline 1.3 & 0.00056 & 0.00001 & 0.00000 & 0.00000 & 0.00000 & 0.00000 & 0.00000 & 0.00000 & 0 \\
\hline 1.4 & 0.00387 & 0.00016 & 0.00001 & 0.00000 & 0.00000 & 0.00000 & 0.00000 & 0.00000 & 0 \\
\hline 1.5 & 0.01565 & 0.00161 & 0.00014 & 0.00001 & 0.00000 & 0.00000 & 0.00000 & 0.00000 & 0 \\
\hline 1.6 & 0.04357 & 0.00979 & 0.00181 & 0.00030 & 0.00005 & 0.00001 & 0.00000 & 0.00000 & 0 \\
\hline 1.7 & 0.08592 & 0.03889 & 0.01378 & 0.00438 & 0.00131 & 0.00038 & 0.00011 & 0.00000 & 0 \\
\hline 1.8 & 0.10875 & 0.09702 & 0.06302 & 0.03555 & 0.01857 & 0.00925 & 0.00446 & 0.00020 & 0 \\
\hline 1.9 & 0.05763 & 0.10669 & 0.12612 & 0.12479 & 0.11169 & 0.09373 & 0.07524 & 0.02433 & 0 \\
\hline
\end{tabular}

TABLE XVI: Residual mass at $\beta=6.0$ for some odd $N_{s}$, in Landau gauge.

\begin{tabular}{|l|rrrrrr|}
\hline \hline$M$ & $N_{s}=13$ & $N_{s}=14$ & $N_{s}=15$ & $N_{s}=16$ & $N_{s}=17$ & $N_{s}=18$ \\
\hline 0.1 & -0.211 & -0.206 & -0.201 & -0.195 & -0.188 & -0.181 \\
0.2 & -0.132 & -0.114 & -0.099 & -0.085 & -0.072 & -0.062 \\
0.3 & -0.043 & -0.033 & -0.025 & -0.018 & -0.014 & -0.010 \\
0.4 & -0.009 & -0.006 & -0.004 & -0.002 & -0.002 & -0.001 \\
0.5 & -0.001 & -0.001 & 0.000 & 0.000 & 0.000 & 0.000 \\
0.6 & 0.000 & 0.000 & 0.000 & 0.000 & 0.000 & 0.000 \\
0.7 & 0.000 & 0.000 & 0.000 & 0.000 & 0.000 & 0.000 \\
0.8 & 0.000 & 0.000 & 0.000 & 0.000 & 0.000 & 0.000 \\
0.9 & 0.000 & 0.000 & 0.000 & 0.000 & 0.000 & 0.000 \\
1.0 & 0.000 & 0.000 & 0.000 & 0.000 & 0.000 & 0.000 \\
1.1 & 0.000 & 0.000 & 0.000 & 0.000 & 0.000 & 0.000 \\
1.2 & 0.000 & 0.000 & 0.000 & 0.000 & 0.000 & 0.000 \\
1.3 & 0.000 & 0.000 & 0.000 & 0.000 & 0.000 & 0.000 \\
1.4 & 0.000 & 0.000 & 0.000 & 0.000 & 0.000 & 0.000 \\
1.5 & -0.001 & 0.000 & 0.000 & 0.000 & 0.000 & 0.000 \\
1.6 & -0.006 & 0.004 & -0.002 & 0.002 & -0.001 & 0.001 \\
1.7 & -0.026 & 0.020 & -0.015 & 0.012 & -0.009 & 0.007 \\
1.8 & -0.074 & 0.067 & -0.060 & 0.053 & -0.047 & 0.041 \\
1.9 & -0.092 & 0.098 & -0.102 & 0.105 & -0.106 & 0.107 \\
\hline \hline
\end{tabular}

while for $M<1.8$ they are increased by renormalization so that the minimum finally ends up located near $M=1.8$.

Notice that for $M=1.9$ the residual mass at $N_{s}=12$ is larger than at $N_{s}=8$, and at $N_{s}=16$ is even larger. We will discuss more in detail this kind of behavior in Sect. VIII

As an aside, we have also investigated what happens when one chooses an odd value of $N_{s}$. As can be seen from Tables XVI and XVII which are representative of the general situation, when $M$ is smaller than 1 the resid-
TABLE XVII: Residual mass at $\beta=5.2$ for some odd $N_{s}$, in Landau gauge.

\begin{tabular}{|r|rrrrrr|}
\hline \hline$M$ & $N_{s}=13$ & $N_{s}=14$ & $N_{s}=15$ & $N_{s}=16$ & $N_{s}=17$ & $N_{s}=18$ \\
\hline 0.1 & -0.236 & -0.231 & -0.226 & -0.219 & -0.212 & -0.205 \\
0.2 & -0.149 & -0.129 & -0.112 & -0.096 & -0.082 & -0.070 \\
0.3 & -0.049 & -0.037 & -0.028 & -0.021 & -0.016 & -0.012 \\
0.4 & -0.011 & -0.007 & -0.004 & -0.003 & -0.002 & -0.001 \\
0.5 & -0.002 & -0.001 & 0.000 & 0.000 & 0.000 & 0.000 \\
0.6 & 0.000 & 0.000 & 0.000 & 0.000 & 0.000 & 0.000 \\
0.7 & 0.000 & 0.000 & 0.000 & 0.000 & 0.000 & 0.000 \\
0.8 & 0.000 & 0.000 & 0.000 & 0.000 & 0.000 & 0.000 \\
0.9 & 0.000 & 0.000 & 0.000 & 0.000 & 0.000 & 0.000 \\
1.0 & 0.000 & 0.000 & 0.000 & 0.000 & 0.000 & 0.000 \\
1.1 & 0.000 & 0.000 & 0.000 & 0.000 & 0.000 & 0.000 \\
1.2 & 0.000 & 0.000 & 0.000 & 0.000 & 0.000 & 0.000 \\
1.3 & 0.000 & 0.000 & 0.000 & 0.000 & 0.000 & 0.000 \\
1.4 & 0.000 & 0.000 & 0.000 & 0.000 & 0.000 & 0.000 \\
1.5 & -0.001 & 0.000 & 0.000 & 0.000 & 0.000 & 0.000 \\
1.6 & -0.006 & 0.004 & -0.003 & 0.002 & -0.001 & 0.001 \\
1.7 & -0.030 & 0.024 & -0.018 & 0.014 & -0.010 & 0.008 \\
1.8 & -0.089 & 0.080 & -0.071 & 0.063 & -0.055 & 0.048 \\
1.9 & -0.114 & 0.120 & -0.124 & 0.126 & -0.127 & 0.127 \\
\hline \hline
\end{tabular}

ual mass has always a negative sign for $N_{s}$ even or odd, but in the more interesting case of $M$ greater than 1 the one-loop residual mass turns out to be positive when $N_{s}$ is even and negative when $N_{s}$ is odd. This could be related to the suggestion which was made by Neuberger some years ago [52], according to which for odd $N_{s}$ the theory which is simulated could correspond to the $\theta=\pi$ regime of QCD.

The residual mass obviously changes also when the coupling $g_{0}$ is varied, as an effect of the loop corrections. Indeed, the overlap between the chiral modes liv- 
TABLE XVIII: Coefficient of $\bar{g}^{2}$ for the quantity $\Delta=Z_{V}-Z_{A}=-\left(Z_{S}-Z_{P}\right) / 2$, which vanishes when chiral symmetry is restored at $N_{s}=\infty$, in Feynman gauge.

\begin{tabular}{|c|c|c|c|c|c|c|c|c|c|}
\hline$M \mid$ & $N_{s}=8$ & $N_{s}=12$ & $N_{s}=16$ & $N_{s}=20$ & $N_{s}=24$ & $N_{s}=28$ & $N_{s}=32$ & $N_{s}=48$ & $N_{s}=\infty$ \\
\hline 0.1 & 6.9399 & 4.4434 & 2.6941 & 1.5749 & 0.8916 & 0.4899 & 0.2622 & 0.0179 & 0 \\
\hline 0.2 & 2.3272 & 0.7211 & 0.1945 & 0.0477 & 0.0110 & 0.0025 & 0.0005 & 0.0000 & 0 \\
\hline 0.3 & 0.5894 & 0.0668 & 0.0064 & 0.0006 & 0.0000 & 0.0000 & 0.0000 & 0.0000 & 0 \\
\hline 0.4 & 0.0994 & 0.0034 & 0.0001 & 0.0000 & 0.0000 & 0.0000 & 0.0000 & 0.0000 & 0 \\
\hline 0.5 & 0.0106 & 0.0001 & 0.0000 & 0.0000 & 0.0000 & 0.0000 & 0.0000 & 0.0000 & 0 \\
\hline 0.6 & 0.0007 & 0.0000 & 0.0000 & 0.0000 & 0.0000 & 0.0000 & 0.0000 & 0.0000 & 0 \\
\hline 0.7 & 0.0000 & 0.0000 & 0.0000 & 0.0000 & 0.0000 & 0.0000 & 0.0000 & 0.0000 & 0 \\
\hline 0.8 & 0.0000 & 0.0000 & 0.0000 & 0.0000 & 0.0000 & 0.0000 & 0.0000 & 0.0000 & 0 \\
\hline 0.9 & 0.0000 & 0.0000 & 0.0000 & 0.0000 & 0.0000 & 0.0000 & 0.0000 & 0.0000 & 0 \\
\hline 1.0 & 0.0000 & 0.0000 & 0.0000 & 0.0000 & 0.0000 & 0.0000 & 0.0000 & 0.0000 & 0 \\
\hline 1.1 & 0.0000 & 0.0000 & 0.0000 & 0.0000 & 0.0000 & 0.0000 & 0.0000 & 0.0000 & 0 \\
\hline 1.2 & 0.0000 & 0.0000 & 0.0000 & 0.0000 & 0.0000 & 0.0000 & 0.0000 & 0.0000 & 0 \\
\hline 1.3 & 0.0000 & 0.0000 & 0.0000 & 0.0000 & 0.0000 & 0.0000 & 0.0000 & 0.0000 & 0 \\
\hline 1.4 & 0.0005 & 0.0000 & 0.0000 & 0.0000 & 0.0000 & 0.0000 & 0.0000 & 0.0000 & 0 \\
\hline 1.5 & 0.0083 & 0.0001 & 0.0000 & 0.0000 & 0.0000 & 0.0000 & 0.0000 & 0.0000 & 0 \\
\hline 1.6 & 0.0701 & 0.0029 & 0.0001 & 0.0000 & 0.0000 & 0.0000 & 0.0000 & 0.0000 & 0 \\
\hline 1.7 & 0.3140 & 0.0501 & 0.0056 & 0.0005 & 0.0000 & 0.0000 & 0.0000 & 0.0000 & 0 \\
\hline 1.8 & 0.8354 & 0.3672 & 0.1288 & 0.0374 & 0.0096 & 0.0022 & 0.0005 & 0.0000 & 0 \\
\hline 1.9 & 2.2075 & 1.5323 & 1.0348 & 0.6904 & 0.4490 & 0.2811 & 0.1686 & 0.0153 & 0 \\
\hline
\end{tabular}

TABLE XIX: Coefficient of $\bar{g}^{2}$ for the part proportional to $1-\alpha$ of $\Delta$.

\begin{tabular}{|r|rrrrrrrrr|}
\hline \hline$M$ & $N_{s}=8$ & $N_{s}=12$ & $N_{s}=16$ & $N_{s}=20$ & $N_{s}=24$ & $N_{s}=28$ & $N_{s}=32$ & $N_{s}=48$ & $N_{s}=\infty \mid$ \\
\hline 0.1 & -1.1115 & -0.8058 & -0.5284 & -0.3257 & -0.1914 & -0.1081 & -0.0590 & -0.0042 & 0 \\
0.2 & -0.4075 & -0.1438 & -0.0416 & -0.0106 & -0.0025 & -0.0006 & -0.0001 & 0.0000 & 0 \\
0.3 & -0.1092 & -0.0140 & -0.0014 & -0.0001 & 0.0000 & 0.0000 & 0.0000 & 0.0000 & 0 \\
0.4 & -0.0191 & -0.0007 & 0.0000 & 0.0000 & 0.0000 & 0.0000 & 0.0000 & 0.0000 & 0 \\
0.5 & -0.0020 & 0.0000 & 0.0000 & 0.0000 & 0.0000 & 0.0000 & 0.0000 & 0.0000 & 0 \\
0.6 & -0.0001 & 0.0000 & 0.0000 & 0.0000 & 0.0000 & 0.0000 & 0.0000 & 0.0000 & 0 \\
0.7 & 0.0000 & 0.0000 & 0.0000 & 0.0000 & 0.0000 & 0.0000 & 0.0000 & 0.0000 & 0 \\
0.8 & 0.0000 & 0.0000 & 0.0000 & 0.0000 & 0.0000 & 0.0000 & 0.0000 & 0.0000 & 0 \\
0.9 & 0.0000 & 0.0000 & 0.0000 & 0.0000 & 0.0000 & 0.0000 & 0.0000 & 0.0000 & 0 \\
1.0 & 0.0000 & 0.0000 & 0.0000 & 0.0000 & 0.0000 & 0.0000 & 0.0000 & 0.0000 & 0 \\
1.1 & 0.0000 & 0.0000 & 0.0000 & 0.0000 & 0.0000 & 0.0000 & 0.0000 & 0.0000 & 0 \\
1.2 & 0.0000 & 0.0000 & 0.0000 & 0.0000 & 0.0000 & 0.0000 & 0.0000 & 0.0000 & 0 \\
1.3 & 0.0000 & 0.0000 & 0.0000 & 0.0000 & 0.0000 & 0.0000 & 0.0000 & 0.0000 & 0 \\
1.4 & -0.0001 & 0.0000 & 0.0000 & 0.0000 & 0.0000 & 0.0000 & 0.0000 & 0.0000 & 0 \\
1.5 & -0.0022 & 0.0000 & 0.0000 & 0.0000 & 0.0000 & 0.0000 & 0.0000 & 0.0000 & 0 \\
1.6 & -0.0186 & -0.0008 & 0.0000 & 0.0000 & 0.0000 & 0.0000 & 0.0000 & 0.0000 & 0 \\
1.7 & -0.0879 & -0.0134 & -0.0015 & -0.0001 & 0.0000 & 0.0000 & 0.0000 & 0.0000 & 0 \\
1.8 & -0.2750 & -0.1064 & -0.0353 & -0.0100 & -0.0025 & -0.0006 & -0.0001 & 0.0000 & 0 \\
1.9 & -0.9106 & -0.5678 & -0.3473 & -0.2140 & -0.1314 & -0.0790 & -0.0461 & -0.0040 & 0 \\
\hline \hline
\end{tabular}

ing near the two walls depends also on the strength of the gauge coupling, and for strong couplings this overlap tends to acquire some nonnegligible values. Furthermore, the residual mass explicitly depends also on the value of the lattice spacing $a$, as can be seen from Eq. (48), since all the numbers presented in the Tables are given, as elsewhere in the paper, in lattice units. The residual mass is thus different for quenched and unquenched simulations made at the same lattice spacing, because $a$ and $g_{0}$ are related in a different way. Let us now consider some typical values of the parameters at which simulations are cur- rently performed. We first fix $M=1.8$ and $N_{s}=16$. In the quenched case we can then take $\beta=6.0$, which corresponds to a lattice spacing of about $2 \mathrm{GeV}$, and according to our one-loop calculations this gives $a m_{\text {res }}=0.05207$ in Feynman gauge and $a m_{\text {res }}=0.05326$ in Landau gauge, that is $m_{\text {res }} \sim 104 \mathrm{MeV}$ and $m_{\text {res }} \sim 107 \mathrm{MeV}$ respectively. The dependence on the gauge seems thus from the numerical point of view not to be very significant. Notice also that if $m_{r e s}^{(0)}$ in Eq. 39 were positive, the values of $m_{\text {res }}^{(1)}$ would be even larger. In the unquenched case, if we take $\beta=5.2$, which corresponds roughly to 
TABLE XX: Coefficient of $\bar{g}^{2}$ for the coefficient of the power-divergent mixing of $O_{d_{1}}, c_{m i x}$ in Eq. (75), in Feynman gauge.

\begin{tabular}{|c|c|c|c|c|c|c|c|c|c|}
\hline$M$ & $N_{s}=8$ & $N_{s}=12$ & $N_{s}=16$ & $N_{s}=20$ & $N_{s}=24$ & $N_{s}=28$ & $N_{s}=32$ & $N_{s}=48$ & $N_{s}=\infty$ \\
\hline 0.1 & -14.1244 & -8.4448 & -5.3682 & -3.4884 & -2.2846 & -1.4996 & -0.9848 & -0.1829 & 0 \\
\hline 0.2 & -5.8166 & -2.4014 & -0.9910 & -0.4075 & -0.1674 & -0.0688 & -0.0283 & -0.0008 & 0 \\
\hline 0.3 & -2.4733 & -0.6070 & -0.1475 & -0.0359 & -0.0087 & -0.0021 & -0.0005 & 0.0000 & 0 \\
\hline 0.4 & -0.9361 & -0.1265 & -0.0169 & -0.0022 & -0.0003 & 0.0000 & 0.0000 & 0.0000 & 0 \\
\hline 0.5 & -0.3094 & -0.0213 & -0.0014 & -0.0001 & 0.0000 & 0.0000 & 0.0000 & 0.0000 & 0 \\
\hline 0.6 & -0.0893 & -0.0029 & -0.0001 & 0.0000 & 0.0000 & 0.0000 & 0.0000 & 0.0000 & 0 \\
\hline 0.7 & -0.0231 & -0.0004 & 0.0000 & 0.0000 & 0.0000 & 0.0000 & 0.0000 & 0.0000 & 0 \\
\hline 0.8 & -0.0059 & -0.0001 & 0.0000 & 0.0000 & 0.0000 & 0.0000 & 0.0000 & 0.0000 & 0 \\
\hline 0.9 & -0.0015 & 0.0000 & 0.0000 & 0.0000 & 0.0000 & 0.0000 & 0.0000 & 0.0000 & 0 \\
\hline 1.0 & 0.0000 & 0.0000 & 0.0000 & 0.0000 & 0.0000 & 0.0000 & 0.0000 & 0.0000 & 0 \\
\hline 1.1 & 0.0008 & 0.0000 & 0.0000 & 0.0000 & 0.0000 & 0.0000 & 0.0000 & 0.0000 & 0 \\
\hline 1.2 & 0.0014 & 0.0000 & 0.0000 & 0.0000 & 0.0000 & 0.0000 & 0.0000 & 0.0000 & 0 \\
\hline 1.3 & 0.0031 & 0.0001 & 0.0000 & 0.0000 & 0.0000 & 0.0000 & 0.0000 & 0.0000 & 0 \\
\hline 1.4 & 0.0127 & 0.0003 & 0.0000 & 0.0000 & 0.0000 & 0.0000 & 0.0000 & 0.0000 & 0 \\
\hline 1.5 & 0.0546 & 0.0029 & 0.0002 & 0.0000 & 0.0000 & 0.0000 & 0.0000 & 0.0000 & 0 \\
\hline 1.6 & 0.1969 & 0.0207 & 0.0024 & 0.0003 & 0.0000 & 0.0000 & 0.0000 & 0.0000 & 0 \\
\hline 1.7 & 0.5774 & 0.1129 & 0.0229 & 0.0049 & 0.0011 & 0.0002 & 0.0001 & 0.0000 & 0 \\
\hline 1.8 & 1.3844 & 0.4817 & 0.1715 & 0.0615 & 0.0224 & 0.0083 & 0.0031 & 0.0001 & 0 \\
\hline 1.9 & 2.9825 & 1.5917 & 0.9340 & 0.5665 & 0.3480 & 0.2148 & 0.1328 & 0.0198 & 0 \\
\hline
\end{tabular}

TABLE XXI: Coefficient of $\bar{g}^{2}$ for the part proportional to $1-\alpha$ of $c_{m i x}$.

\begin{tabular}{|r|rrrrrrrrr}
\hline \hline$M$ & $N_{s}=8$ & $N_{s}=12$ & $N_{s}=16$ & $N_{s}=20$ & $N_{s}=24$ & $N_{s}=28$ & $N_{s}=32$ & $N_{s}=48$ & $N_{s}=\infty$ \\
\hline 0.1 & 0.3241 & 0.1056 & 0.0395 & 0.0162 & 0.0071 & 0.0033 & 0.0015 & 0.0001 \\
0.2 & 0.0480 & 0.0093 & 0.0019 & 0.0004 & 0.0001 & 0.0000 & 0.0000 & 0.0000 \\
0.3 & 0.0093 & 0.0008 & 0.0001 & 0.0000 & 0.0000 & 0.0000 & 0.0000 & 0.0000 \\
0.4 & 0.0014 & 0.0000 & 0.0000 & 0.0000 & 0.0000 & 0.0000 & 0.0000 & 0.0000 \\
0.5 & 0.0001 & 0.0000 & 0.0000 & 0.0000 & 0.0000 & 0.0000 & 0.0000 & 0.0000 \\
0.6 & 0.0000 & 0.0000 & 0.0000 & 0.0000 & 0.0000 & 0.0000 & 0.0000 & 0.0000 \\
0.7 & 0.0000 & 0.0000 & 0.0000 & 0.0000 & 0.0000 & 0.0000 & 0.0000 & 0.0000 \\
0.8 & 0.0000 & 0.0000 & 0.0000 & 0.0000 & 0.0000 & 0.0000 & 0.0000 & 0.0000 \\
0.9 & 0.0000 & 0.0000 & 0.0000 & 0.0000 & 0.0000 & 0.0000 & 0.0000 & 0.0000 \\
1.0 & 0.0000 & 0.0000 & 0.0000 & 0.0000 & 0.0000 & 0.0000 & 0.0000 & 0.0000 \\
1.1 & 0.0000 & 0.0000 & 0.0000 & 0.0000 & 0.0000 & 0.0000 & 0.0000 & 0.0000 \\
1.2 & 0.0000 & 0.0000 & 0.0000 & 0.0000 & 0.0000 & 0.0000 & 0.0000 & 0.0000 \\
1.3 & 0.0000 & 0.0000 & 0.0000 & 0.0000 & 0.0000 & 0.0000 & 0.0000 & 0.0000 \\
1.4 & 0.0000 & 0.0000 & 0.0000 & 0.0000 & 0.0000 & 0.0000 & 0.0000 & 0.0000 \\
1.5 & -0.0001 & 0.0000 & 0.0000 & 0.0000 & 0.0000 & 0.0000 & 0.0000 & 0.0000 \\
1.6 & -0.0013 & 0.0000 & 0.0000 & 0.0000 & 0.0000 & 0.0000 & 0.0000 & 0.0000 \\
1.7 & -0.0060 & -0.0007 & -0.0001 & 0.0000 & 0.0000 & 0.0000 & 0.0000 & 0.0000 \\
1.8 & 0.0035 & -0.0043 & -0.0015 & -0.0004 & -0.0001 & 0.0000 & 0.0000 & 0.0000 \\
1.9 & 0.4015 & 0.0982 & 0.0218 & 0.0029 & -0.0011 & -0.0013 & -0.0009 & -0.0001 \\
\hline \hline
\end{tabular}

the same lattice spacing of $2 \mathrm{GeV}$ but now to a different bare coupling, we then obtain $a m_{r e s}=0.06164$ in Feynman gauge and $a m_{\text {res }}=0.06302$ in Landau gauge, that is $m_{\text {res }} \sim 123 \mathrm{MeV}$ and $m_{\text {res }} \sim 126 \mathrm{MeV}$ respectively. Results from dynamical domain-wall simulations show that the residual mass in full QCD assumes much higher values than in the quenched case. Dynamical domain-wall fermions present then a larger explicit chiral symmetry breaking. We can infer a similar effect which goes in the same direction from our one-loop perturbative results, although here the difference between quenched and full QCD is not as pronounced as it has been observed in the simulations, suggesting that higher-order corrections and especially nonperturbative effects presumably play a significant rôle.

We have automated the calculations presented in this article by developing suitable FORM codes [53], which are a sizeable extension of the programs which were used in the calculations presented in Ref. [40] for $N_{s}=\infty$. These codes are now able to compute matrix elements for general values of $N_{s}$ and $M$.

The integrals of the Feynman diagrams have been numerically evaluated by keeping $N_{s}$ fixed while at the same time refining the integration grid in the usual four- 
dimensional momentum space (where we needed to consider values of $L_{x}=120$ ). One might wonder whether this way of performing the computation of the integrals, which amounts to taking a finite extra dimension with a small number of points while the number of points in the other four dimensions goes to infinity, is legitimate. One could after all object that the number of lattice points in the standard four dimensions is also bound to be finite in any Monte Carlo simulation (although it can reach the order of $10^{2}$ for each of the coordinates, a number certainly larger than any at present practical value of $N_{s}$ ).

We can however observe that in the usual fourdimensional momentum space one does not encounter so wide variations for the Feynman integrand functions as instead is the case in the fifth dimension, where the Feynman integrands contain functions with an exponential behavior, responsible for the fact that for small $N_{s}$ (of the order of ten) large deviations of the integrals from their asymptotic values can be observed. Indeed, refining the integration grid in the usual four-dimensional momentum space from, say, $L_{x}=60$ to $L_{x}=80$, generally produces minimal differences in the momentum integrals, and it is only in order to extract five or more significant digits that one needs to use $L_{x}=100$ or higher. I think that it is important in this regard to keep in mind that the shape of the propagator in the fifth dimension corresponds to physical effects strictly connected with chirality (or lack thereof), whereas in 4-dimensional momentum space it corresponds to finite size effects only. One could also take the point of view in which a lattice spacing $a_{s}$ for the fifth dimension, which is distinct from the four-dimensional lattice spacing $a$, is introduced, and imagine a situation where one keeps $a_{s}$ finite while the usual $a$ goes to zero.

In the domain-wall perturbative calculations for infinite $N_{s}$ made so far it was implicitly assumed that one could take the limit $N_{s} \rightarrow \infty$ for the quark propagators before actually using them to perform the computation of the Feynman diagrams and the momentum integration. This could in principle present some problems, because these asymptotic propagators know about the AtiyahSinger index, which is something which is not present in the theory at finite $N_{s}$, and furthermore they possess extra infrared singularities because of the exactly massless chiral mode. In the present work we always in the first place compute the Feynman diagrams and the integrals using the exact Feynman rules appropriate for finite $N_{s}$, and only afterwards we try to investigate the limit $N_{s} \rightarrow \infty$ by computing the integrals anew for each increasing value of $N_{s}$. We can then observe that, apart from extreme values of $M$ (see Sect. VIII), these integrals rapidly approach the values obtained with the theory which uses the $N_{s}=\infty$ propagators. By using the exact theory with finite $N_{s}$, we have hence also provided the check that in the large $N_{s}$ limit one indeed recovers the results obtained using the simpler asymptotic theory. We can thus confirm, at least numerically, that the inversion of the limits was legitimate.

In the case of the contribution of the half-circle di- agram of the self-energy to $\Sigma_{0}$, we have also repeated the whole calculation (in a general covariant gauge) by hand, including the evaluation of the gamma algebra and the explicit computation of the sums over the fifth-dimensional indices. The final expressions are very lengthy and it is of no interest to report them here, however this alternative procedure provides a rather independent check of our calculations with respect to both the analytic part and the precision of the numerical integration in the 6-dimensional code. Furthermore, since this time we compute the sums in the extra dimension analytically, this saves 2 dimensions in the numerical integration, which is reduced to the usual 4 , and this is why we are able to give the results for $\Sigma_{0}$ with more precision than otherwise. Of course we cannot always employ this procedure, given that the other diagrams are in general way too complicated for a computation by hand. But this alternative 4-dimensional code for $\Sigma_{0}$ provides another advantage because the dependence on $N_{s}$ is now computed exactly and the computational cost is the same for every $N_{s}$, and thus we can think of getting results also at higher values of $N_{s}$, which would be too expensive in the 6-dimensional code where the computational cost grows as $N_{s}^{2}$.

We conclude this Section by reminding that the tadpoles of course can be calculated for any $N_{s}$ and $M$ with an extremely high precision, which is limited only by the knowledge of $Z_{0}$ (at present known with about 400 digits [51]).

\section{BILINEAR DIFFERENCES}

We now consider the calculation at finite $N_{s}$ of matrix elements of some operators. In this Section we present one-loop results for the (finite) differences of chirallyrelated bilinear operators, which should become zero at infinite $N_{s}$, that is when chiral symmetry is fully restored. Since the vector and axial-vector currents renormalize differently when chiral symmetry is broken, an estimate of chirality-breaking effects can indeed be given by how much for a given finite $N_{s}$ the perturbative results for these currents differ from each other. The quantity $\Delta=Z_{V}-Z_{A}$ provides such a measure of chirality breaking. Moreover, one finds that $Z_{V}-Z_{A}=-\left(Z_{S}-Z_{P}\right) / 2$ [54]. The fact that we obtain for $\Delta$ the same number with a very good precision whether we consider the vector and axial-vector case or the scalar and pseudoscalar case then provides a compelling check of our calculations.

We can successfully obtain all results for $\Delta$ by computing only finite lattice diagrams, because of the following three facts. For one thing, the anomalous dimensions of the operators which are chirally related are the same also when one considers the subleading orders (see Sect. IV]) that is $\gamma_{V}^{\left(N_{s}, M\right)}=\gamma_{A}^{\left(N_{s}, M\right)}$ and $\gamma_{S}^{\left(N_{s}, M\right)}=\gamma_{P}^{\left(N_{s}, M\right)}$. Furthermore, the continuum values of the finite parts, called $R^{\overline{\mathrm{MS}}}$ in Eq. (64), are also equal for these pairs of operators. Finally, the mismatch between lattice (at fi- 
nite $N_{s}$ ) and continuum anomalous dimensions that we discussed at the end of Sect. IV is also the same for the chirally-related operators, and thus it cancels in their differences. We remind that the vector and axial-vector currents have now a nonvanishing anomalous dimension, which becomes zero only when $N_{s}=\infty$.

As we can gather in Tables XVIII and XIX the decrease of the amount of chirality breaking connected to $\Delta$ follows a pattern similar to the one that we have encountered in the case of the critical mass and to the one that we will see for the operator discussed in the next Section, that is $\Delta$ is rather large for small $N_{s}$ and large $|1-M|$, and decreases when $N_{s}$ grows or when $|1-M|$ tends towards zero. Again, the part proportional to $(1-\alpha)$ of $\Delta$ shows a violation of gauge invariance, since if gauge invariance were respected these numbers would have to be zero, as it happens for Wilson or overlap fermions. We can notice that here the deviations from gauge invariance are of the same order as the ones for the critical mass, and that they are small. The operator studied in the next Section gives also very similar results regarding this point.

Taking a typical case, where $M=1.8$ and $N_{s}=16$ and $a^{-1} \sim 2 \mathrm{GeV}$, the numbers that we have obtained imply for QCD a chiral violation of about $2 \mathrm{MeV}$, with the quenched case giving the slightly lower value. These numbers are much smaller, at given $M$ and $N_{s}$, than the ones obtained for $m_{\text {res }}$. This could mean that the chiral violations are here of $O\left(m_{r \rho s}^{2}\right)$, as it has been suggested for other quantities in [17, 42].

It is also interesting to compare the numbers presented in this Section to the results that one obtains for this quantity when Wilson fermions are used. In this case one gets $\Delta=4.82152$ for the unimproved case and $\Delta=$ 1.53633 for the improved case, which correspond to chiral violations which are at least of order $30 \mathrm{MeV}$ for $\beta \sim 6.0$.

\section{A POWER-DIVERGENT MIXING}

We think that it is also very instructive to study the case of an operator mixing which gets completely suppressed only when chiral symmetry is fully operative, so that the nonzero amount of residual mixing present for any given finite $N_{s}$ provides another quantitative measure of chiral violations. One of the simplest examples of this kind of mixings is probably furnished by the operator

$$
O_{d_{1}}=\bar{q} \gamma_{[4} \gamma_{5} D_{1]} q
$$

which taken together with $O_{a_{2}, d}=\bar{q} \gamma_{\{1} \gamma_{5} D_{4\}} q$ (or, in an alternative representation of the hypercubic group, $\left.O_{a_{2}, e}=\bar{q} \gamma_{4} \gamma_{5} D_{4} q-\frac{1}{3} \sum_{i=1}^{3} \bar{q} \gamma_{i} \gamma_{5} D_{i} q\right)$, determines the first moment of the $g_{2}$ structure function, which measures the distribution of the (chiral even) transverse spin of quarks inside hadrons, and also receives contributions from twist-3 operators. More details on these operators can be found in 55, 56] (of which we follow the notation) and references therein. Matrix elements of the op- erator $O_{d_{1}}$ have been recently simulated using quenched domain-wall fermions with the DBW2 gauge action [18]. We remind that the symbol [] denotes antisymmetrization over the relevant Lorentz indices, while \{\} denotes symmetrization, and that for the covariant derivatives $D=\vec{D}-\overleftarrow{D}$ we use the lattice discretizations

$$
\begin{gathered}
\vec{D}_{\mu} q(x)=\frac{1}{2}\left[U_{\mu}(x) q(x+\hat{\mu})-U_{\mu}^{\dagger}(x-\hat{\mu}) q(x-\hat{\mu})\right] \\
\bar{q}(x) \stackrel{\leftarrow}{D_{\mu}}=\frac{1}{2}\left[\bar{q}(x+\hat{\mu}) U_{\mu}^{\dagger}(x)-\bar{q}(x-\hat{\mu}) U_{\mu}(x-\hat{\mu})\right] .
\end{gathered}
$$

We point out that many of the mixings which one encounters in the study of physical processes, like for instance the ones occurring in the renormalization of the second moment of the unpolarized parton distribution [57, 58], arise as a consequence of the breaking of Lorentz symmetry (or, in other cases, of other symmetries apart from chirality) and hence do not interest us much in the present context. The power-divergent mixing on the lattice of $O_{d_{1}}$ with an operator of lower dimension, which can be written as

$$
c_{m i x} \cdot \frac{i}{a} \bar{q} \sigma_{41} \gamma_{5} q
$$

is instead particularly interesting because is only caused by the breaking of chirality, and hence it provides a quantitative measure of how much chiral symmetry has been broken. The finite coefficient $c_{m i x}$ must vanish for infinite $N_{s}$, when chiral symmetry is fully restored, and $O_{d_{1}}$ becomes in this case multiplicatively renormalized (like it also is when overlap fermions are used). For finite $N_{s}$ the values of this coefficient in the Feynman gauge are reported in Table $\mathrm{XX}$, where we can see that in general this mixing can be considered to be almost negligible. Were this not the case, the removal of these lattice artifacts in Monte Carlo simulations would become quite challenging.

The results show that large chiral violations are present only for very small $N_{s}$, or when $M$ is rather close to 0 or 2. Taking a typical case, for $M=1.8$ and $N_{s}=16$, and $a^{-1} \sim 2 \mathrm{GeV}$, the chiral violations come out of about $3 \mathrm{MeV}$, with the quenched case giving the slightly lower value. These numbers are much smaller, at given $M$ and $N_{s}$, than the ones obtained for $m_{r e s}$. It could be that, as it has been suggested for other quantities in [17, 42], the chiral violations are here quadratic in $m_{r e s}$, that is this coefficient is doubly suppressed.

Again, as can be deduced from Table XXI gauge invariance is lost in the theory at finite $N_{s}$. The part proportional to $1-\alpha$ of $c_{m i x}$ vanishes only when $N_{s}=\infty$ (and this happens also when for example Wilson or overlap fermions are used).

Wilson fermions also suffer from this power-divergent mixing which is caused by the breaking of chirality, but the mixing coefficient is in this case gauge invariant. However, it takes the values $c_{m i x}=16.243762$ for the 
unimproved and $c_{m i x}=8.798732$ for the improved case with $c_{s w}=1$, so that compared to domain-wall fermions at the standard choices of $N_{s}$ and $M$, the Wilson violations are then about two orders of magnitude higher. For overlap fermions of course $c_{m i x}$ is zero.

\section{TOWARD THE BORDERS}

It it also interesting to see what happens when the domain-wall height $M$ is chosen dangerously close to 0 or 2 , that is at the edges of its (at lowest order) allowed values. After investigating in various cases, we have found as a general phenomenon that the exponential decay in $N_{s}$ of the chiral violations slows down when approaching these borders. In some instances it can even happen that for a fixed $M$ chosen very near to one of the edges the chiral violations increase with $N_{s}$, at least up to a certain point.

One can observe this behavior already by looking at the tadpole diagrams, which can be calculated exactly. For $M \rightarrow 0$ they assume in fact the asymptotic expressions (in Feynman gauge)

$$
\frac{4}{3} M T_{d}\left(N_{s}+3+\frac{2}{N_{s}}\right)
$$

in the case of $\Sigma_{0}$, and

$$
\frac{1}{3} M T_{d}\left(2 N_{s}+3+\frac{1}{N_{s}}\right)
$$

in the case of $\Sigma_{1}$. For $M \rightarrow 2$ one just takes into account the fact that these tadpoles are respectively odd and even upon reflection around the point $M=1$. We can then immediately see that for a given $N_{s}$ the tadpoles tend to zero when $M$ approaches 0 or 2 . From Table VI we can also see that the decay in $N_{s}$ (at a constant $M$ ) of the tadpole of $\Sigma_{0}$ tends to become slower and slower as $M$ nears the borders, until the rate of decay probably vanishes at some point and the tadpole then reaches the asymptotic regime of Eq. (76), where actually the chiral violations grow with $N_{s}$ (at fixed $M$ ). We can observe a similar behavior also for the other quantities considered in this article, which we have run for values of $M$ close to $|1-M| \sim 0.99$ and $|1-M| \sim 0.999$. For $\Delta$ and $c_{m i x}$ the rate of the exponential decay in $N_{s}$ keeps decreasing when one approaches the borders, until a likely final disappearance, even though these quantities instead increase in $M$ (for a fixed $N_{s}$ ) when $M \rightarrow 0$ and $M \rightarrow 2$. In fact, $c_{m i x}$ tends to diverge very fast when $M \rightarrow 0$.

The slowing of the exponential decays of $m_{\text {res }}, \Delta$ and $c_{\text {mix }}$ when one moves $M$ such that $|1-M| \rightarrow 1$ can be related to the decrease of the mobility edge $\lambda_{c}$ towards zero in these extreme regions of $M$ [43, 44, 45]. Choosing a value of $M$ too close to 0 or 2 can then become dangerous, because the mobility edge has to remain well above zero in order to perform reliable Monte Carlo simulations, otherwise the restoration of chiral symmetry can become problematic. The fall of the mobility edge to zero signals the onset of the Aoki phase, and this can be pictorially seen for example in Fig. 1 of Ref. 43] or [45], where in fact it corresponds to moving, for $g_{0}$ not too large, from the rightmost $C$ phase towards the $B$ phase, which one eventually enters through one of the thin "fingers".

We can thus briefly sum up the behavior of the chiral violations when $M$ changes as follows. The coefficient of their exponential decay is zero or rather small when $M$ is in the vicinity of 1 . It then grows when $M$ moves towards either 0 or 2, before decreasing again and at last getting rather small for $M \sim 0$ and $M \sim 2$.

A remarkable thing that we have observed for values of $M$ very near the edges is that $m_{r e s}$ and $\Delta$ happen to be smaller for small $N_{s}$ than for larger values of $N_{s}$, that is the chiral violation initially grow in $N_{s}$ instead of decaying exponentially. This observed initial growth is just a temporary one, before eventually $m_{\text {res }}$ or $\Delta$ starts to decay as expected. But it could be that eventually a behavior like the one of Eqs. (76) and (77) sets in, that is the exponential decay disappears altogether and one can only see a steady increase with $N_{s}$.

For $c_{\text {mix }}$ this does not seem to happen, that is we always observe a clear exponential-like decay, without any initial growth in $N_{s}$, at least for $N_{s} \geq 8$ and for the values of $M$ that we have investigated, up to $2-M=10^{-10}$.

Numerical examples that illustrate this phenomenon of the initial growth in $N_{s}$ can be seen in the case of $m_{r e s}$ in Tables XXII and XXIII which refer to $M \rightarrow 2$ (similar things can also be seen for the other limit, $M \rightarrow 0$ ). The onset of this behavior takes place around $M=1.9$, as can also be observed in the last line of Tables XII to XV Furthermore, we can notice that the exponential decay which follows these regions of initial growth in $N_{s}$ sets in at ever higher values of $N_{s}$ when one gets nearer and nearer to 2 (note that for $M>1.95$ the maximum of $m_{\text {res }}$ is reached for $N_{s}>28$ ). We can then speak of a sort of suppression of the chiral violations for small $N_{s}$, which becomes stronger as $M$ approaches the borders, and it could be that in this case the density of eigenvalues or the radius of the modes in Eq. (1) change in such a way to produce this kind of effect, or that this equation breaks down in this region. It could also happen that the value of $N_{s}$ for which the chiral violations reach their maximum (at fixed $M$ ) is further and further shifted toward higher values of $N_{s}$ until one cannot observe any exponential decay at all even for very large $N_{s}$, and possibly for all $N_{s}$, as in the exact asymptotic results of Eqs. (76) and (77).

In Tables XXIV and XXV we can observe a similar behavior in the case of the difference of the vector and axial-vector currents. Since $c_{m i x}$ apparently does not instead show these effects, it could be that this quantity is described by a somewhat different formula than Eq. (1).

It would be interesting to carry out further studies about these phenomena which happen near the borders of $M$, and more investigations in the future could clarify these issues. We have however seen that already at one 
loop we can observe interesting features, in some cases corresponding to what is expected from general considerations which can be derived from other methods.

TABLE XXII: The residual mass near $M=2$, at $\beta=5.2$ in Feynman gauge.

\begin{tabular}{|c|cccccc|}
\hline \hline$M$ & $N_{s}=8$ & $N_{s}=12$ & $N_{s}=16$ & $N_{s}=20$ & $N_{s}=24$ & $N_{s}=28$ \\
\hline 1.91 & 0.043 & 0.094 & 0.120 & 0.127 & 0.120 & 0.107 \\
1.92 & 0.034 & 0.085 & 0.115 & 0.128 & 0.128 & 0.120 \\
1.93 & 0.026 & 0.074 & 0.106 & 0.125 & 0.132 & 0.130 \\
1.94 & 0.018 & 0.061 & 0.094 & 0.117 & 0.130 & 0.134 \\
1.95 & 0.011 & 0.048 & 0.080 & 0.104 & 0.121 & 0.132 \\
\hline \hline
\end{tabular}

TABLE XXIII: The residual mass near $M=2$, at $\beta=5.2$ in Landau gauge.

\begin{tabular}{|c|crrrrr|}
\hline \hline$M$ & $N_{s}=8$ & $N_{s}=12$ & $N_{s}=16$ & $N_{s}=20$ & $N_{s}=24$ & $N_{s}=28$ \\
\hline 1.91 & 0.049 & 0.099 & 0.124 & 0.130 & 0.122 & 0.108 \\
1.92 & 0.040 & 0.089 & 0.119 & 0.131 & 0.130 & 0.122 \\
1.93 & 0.031 & 0.078 & 0.110 & 0.128 & 0.134 & 0.132 \\
1.94 & 0.023 & 0.065 & 0.098 & 0.120 & 0.132 & 0.137 \\
1.95 & 0.015 & 0.052 & 0.083 & 0.107 & 0.124 & 0.134 \\
\hline \hline
\end{tabular}

TABLE XXIV: Coefficient of $\bar{g}^{2}$ for $\Delta$ near $M=2$, in Feynman gauge.

\begin{tabular}{|r|rrrrrr|}
\hline \hline$M$ & $N_{s}=8$ & $N_{s}=12$ & $N_{s}=16$ & $N_{s}=20$ & $N_{s}=24$ & $N_{s}=28$ \\
\hline 1.94 & 3.699 & 3.151 & 2.488 & 1.915 & 1.467 & 1.125 \\
1.95 & 4.246 & 3.858 & 3.220 & 2.594 & 2.063 & 1.637 \\
1.96 & 4.885 & 4.753 & 4.220 & 3.592 & 2.995 & 2.475 \\
1.97 & 5.630 & 5.883 & 5.586 & 5.063 & 4.474 & 3.897 \\
1.98 & 6.494 & 7.301 & 7.442 & 7.225 & 6.823 & 6.332 \\
1.99 & 7.495 & 9.073 & 9.945 & 10.377 & 10.524 & 10.481 \\
\hline \hline
\end{tabular}

\section{CONCLUSIONS}

In this article we have presented the calculation of a few one-loop amplitudes for domain-wall fermions at finite $N_{s}$, with the intention of gathering some knowledge about the extent of chiral symmetry breaking for choices of $N_{s}$ which are far away from the case of infinite extent in the extra fifth dimension. In particular, we have studied three quantities whose deviations from their values at $N_{s}=\infty$ can provide some significant estimates of chiral violations: the residual mass, the difference between the vector and axial-vector renormalization constants, and a power-divergent mixing of a deep-inelastic operator which is entirely due to the breaking of chirality.

We have automated the perturbative calculations by developing suitable FORM codes. We have found that the pattern of the deviations from the case of exact chirality turns out to be approximately the same for all
TABLE XXV: Coefficient of $\bar{g}^{2}$ for $\Delta$ near $M=2$, in Landau gauge.

\begin{tabular}{|r|rrrrrr|}
\hline \hline$M$ & $N_{s}=8$ & $N_{s}=12$ & $N_{s}=16$ & $N_{s}=20$ & $N_{s}=24$ & $N_{s}=28$ \\
\hline 1.94 & 2.112 & 1.897 & 1.558 & 1.237 & 0.973 & 0.763 \\
1.95 & 2.416 & 2.310 & 1.997 & 1.654 & 1.346 & 1.090 \\
1.96 & 2.774 & 2.836 & 2.602 & 2.268 & 1.929 & 1.621 \\
1.97 & 3.193 & 3.503 & 3.432 & 3.179 & 2.856 & 2.522 \\
1.98 & 3.682 & 4.346 & 4.564 & 4.522 & 4.335 & 4.071 \\
1.99 & 4.251 & 5.402 & 6.098 & 6.488 & 6.673 & 6.718 \\
\hline \hline
\end{tabular}

quantities studied, that is these violations substantially increase when $N_{s}$ becomes small or when $M$ approaches 0 or 2 , i.e., close to the borders of the region of allowed values of $M$. Our perturbative calculations show indeed that the numerical deviations from the case of infinite extension in the fifth dimension depend, apart from $N_{s}$ and to a smaller extent from the bare coupling $g_{0}$, very strongly on the choice of $M$. These deviations can become rather pronounced when $M$ is close to the borders of the region of allowed values. For $M=1.8$, a standard choice in Monte Carlo simulations, chiral violations remain still not small for $N_{s}=16$, as can be seen from $m_{\text {res }}$, which for a lattice spacing of $2 \mathrm{GeV}$ is equal to about $100 \mathrm{MeV}$ in the quenched case and about $120 \mathrm{MeV}$ in full QCD. For the difference between the vector and axial-vector renormalization constants as well as for the power-divergent mixing the chiral violations are instead of about 2-3 MeV, suggesting that they are of higher order in $m_{\text {res }}$.

We think that to the extent that one-loop perturbation theory can provide hints to the actual behavior of these quantities, and if higher-order corrections do not strongly compensate for these 1-loop effects, our work shows that chiral violations at $N_{s}=16$ are still somewhat rather pronounced, and it suggests that one should perhaps consider performing simulations which use at least $N_{s}=20$ or $N_{s}=24$ points in the fifth dimension, in order to be able to work with reasonably small chiral violations.

Our work also confirms (though only from the numerical side) the legitimacy of the assumptions made in previous calculations at infinite $N_{s}$, where from the start it was postulated that the limit $N_{s} \rightarrow \infty$ for the quark propagators could be taken before doing the actual computations of the Feynman diagrams and the numerical integration. The asymptotic propagators introduce in fact spurious infrared singularities not present in the theory at finite $N_{s}$, and in passing from $N_{s}=\infty$ to any finite value of $N_{s}$ the analytical Atiyah-Singer index of the Dirac operator, which protects the quarks from acquiring a nonzero mass, disappears. One cannot then be certain that this transition occurs with a smooth and continuous behavior, although at the end it is likely that no problem is present 52]. We can observe that the results which we have presented in this work rapidly approach for large $N_{s}$ the numbers which were obtained with the simplified asymptotic theory, with the exception of the regions very 
close to the borders of allowed values, $M \rightarrow 0$ or $M \rightarrow 2$, where other effects can come into play. We can then a posteriori confirm that, at least for one-loop calculations made at choices of $M$ which are not too extreme, the inversion of the limits was numerically legitimate.

A disturbing finding of our calculations is the pathological behavior of renormalization factors, which are no longer gauge invariant, although the deviations from gauge invariance are not numerically large, even for values of $M$ far away from 1 (the pattern of chiral violations seems to be the same for all quantities considered in this work). It could be that the act of choosing a definite gauge affects the amount of chirality-breaking effects in Monte Carlo simulations as well. Furthermore, the coefficients of the logarithmic divergences turn out to depend on $N_{s}$ and $M$, and only for $N_{s}=\infty$ they are the same as the ones calculated in the continuum. In particular, the anomalous dimension of the vector (as well as the axialvector) current is not zero, and not constant in $N_{s}$ and $M$. It would be interesting in the future to investigate in more detail these phenomena, which could perhaps contribute to a fuller understanding of domain-wall simulations.

\section{Acknowledgments}

I am grateful for the support by Fonds zur Förderung der Wissenschaftlichen Forschung in Österreich (FWF), Project P16310-N08.
[1] D. B. Kaplan, Phys. Lett. B 288, 342 (1992) arXiv:hep-lat/9206013.

[2] Y. Shamir, Nucl. Phys. B 406, 90 (1993) arXiv:hep-lat/9303005.

[3] V. Furman and Y. Shamir, Nucl. Phys. B 439, 54 (1995) arXiv:hep-lat/9405004.

[4] P. H. Ginsparg and K. G. Wilson, Phys. Rev. D 25, 2649 (1982).

[5] M. Lüscher, Phys. Lett. B 428, 342 (1998) arXiv:hep-lat/9802011.

[6] A. Yamada, Phys. Rev. D 57, 1433 (1998) arXiv:hep-lat/9705040.

[7] A. Yamada, Nucl. Phys. B 514, 399 (1998) arXiv:hep-lat/9707032.

[8] Y. Kikukawa, Phys. Rev. D 65, 074504 (2002) arXiv:hep-lat/0105032.

[9] T. Blum and A. Soni, Phys. Rev. D 56, 174 (1997) arXiv:hep-lat/9611030.

[10] T. Blum and A. Soni, Phys. Rev. Lett. 79, 3595 (1997) arXiv:hep-lat/9706023.

[11] S. Aoki, T. Izubuchi, Y. Kuramashi and Y. Taniguchi, Phys. Rev. D 62, 094502 (2000) arXiv:hep-lat/0004003.

[12] P. Chen et al., Phys. Rev. D 64, 014503 (2001) arXiv:hep-lat/0006010.

[13] T. Blum et al., Phys. Rev. D 69, 074502 (2004) arXiv:hep-lat/0007038.

[14] T. Blum et al. [RBC Collaboration], Phys. Rev. D 68, 114506 (2003) arXiv:hep-lat/0110075.

[15] J. I. Noaki et al. [CP-PACS Collaboration], Phys. Rev. D 68, 014501 (2003) arXiv:hep-lat/0108013.

[16] Y. Aoki et al., Phys. Rev. D 69, 074504 (2004) arXiv:hep-lat/0211023.

[17] Y. Aoki et al., Phys. Rev. D 72, 114505 (2005) arXiv:hep-lat/0411006.

[18] K. Orginos, T. Blum and S. Ohta, Phys. Rev. D 73, 094503 (2006) arXiv:hep-lat/0505024.

[19] Y. Aoki et al., Phys. Rev. D 73, 094507 (2006) arXiv:hep-lat/0508011.

[20] J. W. Negele et al. [LHPC Collaboration], Int. J. Mod. Phys. A 21, 720 (2006) arXiv:hep-lat/0509101.

[21] N. Yamada, T. Blum, M. Hayakawa and T. Izubuchi [RBC Collaboration], PoS LAT2005, 092 (2005)
arXiv:hep-lat/0509124.

[22] M. f. Lin, PoS LAT2005, $094 \quad$ (2005) arXiv:hep-lat/0509178.

[23] R. G. Edwards et al. [LHPC Collaboration], PoS LAT2005, 056 (2005) arXiv:hep-lat/0509185.

[24] C. Dawson, T. Izubuchi, T. Kaneko, S. Sasaki and A. Soni, PoS LAT2005, 337 (2005) arXiv:hep-lat/0510018.

[25] J. Noaki, PoS LAT2005, $350 \quad$ (2005) arXiv:hep-lat/0510019.

[26] R. G. Edwards et al. [LHPC Collaboration], Phys. Rev. Lett. 96, 052001 (2006) arXiv:hep-lat/0510062.

[27] S. Ohta, H. Lin and N. Yamada [RBC Collaboration], PoS LAT2005, 096 (2005) arXiv:hep-lat/0510071.

[28] K. Hashimoto, T. Izubuchi and J. Noaki [RBCUKQCD Collaborations], PoS LAT2005, 093 (2005) arXiv:hep-lat/0510079.

[29] F. Berruto, T. Blum, K. Orginos and A. Soni, Phys. Rev. D 73, 054509 (2006) arXiv:hep-lat/0512004.

[30] F. Berruto, T. Blum, K. Orginos and A. Soni, PoS LAT2005, 010 (2005).

[31] H. W. Lin, S. Ohta and N. Yamada [RBC Collaboration], Nucl. Phys. Proc. Suppl. 153, 199 (2006).

[32] P. M. Vranas, Phys. Rev. D 57, 1415 (1998) arXiv:hep-lat/9705023.

[33] P. M. Vranas, Nucl. Phys. Proc. Suppl. 63, 605 (1998) arXiv:hep-lat/9709119.

[34] C. Jung, R. G. Edwards, X. D. Ji and V. Gadiyak, Phys. Rev. D 63, 054509 (2001) arXiv:hep-lat/0007033.

[35] V. G. Gadiyak, Ph.D. Thesis, University of Maryland, UMI-30-78308 (2002).

[36] R. G. Edwards, B. Joo, A. D. Kennedy, K. Orginos and U. Wenger, PoS LAT2005, $146 \quad$ (2005) arXiv:hep-lat/0510086.

[37] S. Aoki and Y. Taniguchi, Phys. Rev. D 59, 054510 (1999) arXiv:hep-lat/9711004.

[38] S. Aoki, T. Izubuchi, Y. Kuramashi and Y. Taniguchi, Phys. Rev. D 59, 094505 (1999) arXiv:hep-lat/9810020.

[39] T. Blum, A. Soni and M. Wingate, Phys. Rev. D 60, 114507 (1999) arXiv:hep-lat/9902016.

[40] S. Capitani, Phys. Rev. D 73, $014505 \quad$ (2006) arXiv:hep-lat/0510091. 
[41] Y. Kikukawa, H. Neuberger and A. Yamada, Nucl. Phys. B 526, 572 (1998) arXiv:hep-lat/9712022.

[42] N. Christ [RBC and UKQCD Collaborations], PoS LAT2005, 345 (2005).

[43] M. Golterman and Y. Shamir, Phys. Rev. D 68, 074501 (2003) arXiv:hep-lat/0306002.

[44] M. Golterman, Y. Shamir and B. Svetitsky, Phys. Rev. D 71, 071502 (2005) arXiv:hep-lat/0407021.

[45] M. Golterman, Y. Shamir and B. Svetitsky, Phys. Rev. D 72, 034501 (2005) arXiv:hep-lat/0503037.

[46] B. Svetitsky, Y. Shamir and M. Golterman, PoS LAT2005, 129 (2005) arXiv:hep-lat/0508015.

[47] D. J. Antonio et al. [RBC and UKQCD Collaborations], PoS LAT2005, 141 (2005).

[48] T. Draper et al., PoS LAT2005, 120 (2005) arXiv:hep-lat/0510075.

[49] R. Narayanan and H. Neuberger, Phys. Lett. B 302, 62 (1993) arXiv:hep-lat/9212019.

[50] G. Martinelli and Y. C. Zhang, Phys. Lett. B 123, 433
(1983)

[51] S. Capitani, Phys. Rept. 382, $113 \quad$ (2003) arXiv:hep-lat/0211036.

[52] H. Neuberger, Phys. Rev. D 57, 5417 (1998) arXiv:hep-lat/9710089.

[53] J. A. M. Vermaseren, arXiv:math-ph/0010025

[54] I acknowledge conversations with Stephan Dürr about the scalar and pseudoscalar correlators.

[55] S. Capitani, Nucl. Phys. B 592, 183 (2001) arXiv:hep-lat/0005008.

[56] S. Capitani, Nucl. Phys. B 597, 313 (2001) arXiv:hep-lat/0009018.

[57] G. Beccarini, M. Bianchi, S. Capitani and G. Rossi, Nucl. Phys. B 456, 271 (1995) arXiv:hep-lat/9506021.

[58] M. Göckeler, R. Horsley, E. M. Ilgenfritz, H. Perlt, P. Rakow, G. Schierholz and A. Schiller, Phys. Rev. D 53, 2317 (1996) arXiv:hep-lat/9508004. 